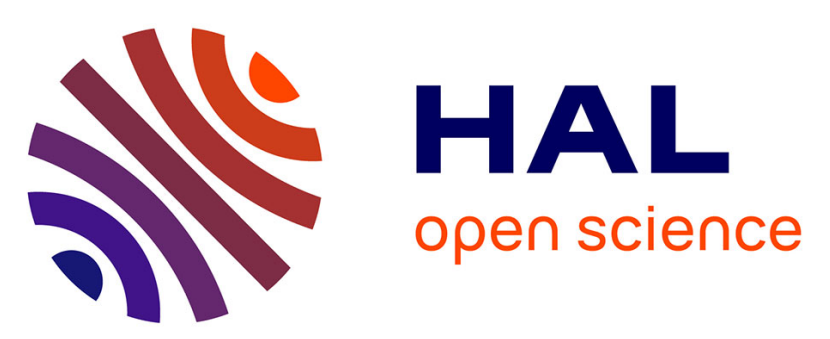

\title{
The thioredoxin encoded by the Rod-derived Cone Viability Factor gene protects cone photoreceptors against oxidative stress
}

Xin Mei, Antoine Chaffiol, Christo Kole, Ying Yang, Géraldine Millet-Puel, Emmanuelle Clérin, Najate Aït-Ali, Jean Bennett, Deniz Dalkara, José-Alain Sahel, et al.

\section{To cite this version:}

Xin Mei, Antoine Chaffiol, Christo Kole, Ying Yang, Géraldine Millet-Puel, et al.. The thioredoxin encoded by the Rod-derived Cone Viability Factor gene protects cone photoreceptors against oxidative stress. Antioxidants and Redox Signaling, 2016, 10.1089/ars.2015.6509 . hal-01297482

\section{HAL Id: hal-01297482 \\ https://hal.sorbonne-universite.fr/hal-01297482}

Submitted on 4 Apr 2016

HAL is a multi-disciplinary open access archive for the deposit and dissemination of scientific research documents, whether they are published or not. The documents may come from teaching and research institutions in France or abroad, or from public or private research centers.
L'archive ouverte pluridisciplinaire HAL, est destinée au dépôt et à la diffusion de documents scientifiques de niveau recherche, publiés ou non, émanant des établissements d'enseignement et de recherche français ou étrangers, des laboratoires publics ou privés. 
Original Research Communication

The thioredoxin encoded by the Rod-derived Cone Viability Factor gene protects cone photoreceptors against oxidative stress

Xin Mei ${ }^{1,2,3}$, Antoine Chaffiol ${ }^{1,2,3}$, Christo Kole ${ }^{1,2,3}$, Ying Yang ${ }^{1,2,3}$, Géraldine Millet-Puel ${ }^{1,2,3}$, Emmanuelle Clérin ${ }^{1,2,3}$, Najate Aït-Ali ${ }^{1,2,3}$, Jean Bennett ${ }^{4}$, Deniz Dalkara ${ }^{1,2,3}$, José-Alain Sahel ${ }^{1,2,3}$, Jens Duebel ${ }^{1,2,3}$ and Thierry Léveillard ${ }^{1,2,3}$

${ }^{1}$ INSERM, U968, Paris, F-75012, France

${ }^{2}$ Sorbonne Universités, UPMC Univ Paris 06, UMR_S 968, Institut de la Vision, Paris, F-75012,

${ }^{3}$ CNRS, UMR_7210, Paris, F-75012, France

${ }^{4}$ Scheie Eye Institute, University of Pennsylvania, Philadelphia, PA, USA

\section{Corresponding author:}

Thierry Léveillard, Department of Genetics, Institut de la Vision, INSERM UMR-S 968, 17, rue Moreau, 75012 Paris, France.

Telephone: 331534625 48; Fax: 33153462502

E-mail: thierry.leveillard@inserm.fr

Word count: 6.25

Reference numbers: 39

Number of greyscale illustrations: 0

Number of color illustrations: 9 


\section{Abstract}

Aims: Rod-derived cone viability factor long (RdCVFL) is an enzymatically active thioredoxin encoded by the nucleoredoxin-like-1 (Nxn/1) gene. The second product of the gene, RdCVF, made by alternative splicing is a novel trophic factor secreted by rods that protects cones in rodent models of retinitis pigmentosa, the most prevalent inherited retinal disease. It acts on the cones by stimulating aerobic glycolysis through its interaction with a complex containing basigin1 and the glucose transport GLUT1. We studied the role of Nxnl1 in cones after its homologous recombination using a transgenic line expressing Cre recombinase under the control of a cone opsin promoter.

Results: We show that the cones of these mice are dysfunctional and degenerate by 8 months of age. The age-related deficit in cones is exacerbated in young animals by exposure to high level of oxygen. In agreement with this phenotype, we found that the cones express only one of the two Nxn/1 gene products, the thioredoxin RdCVFL. Administration of RdCVFL to the mouse carrying a deletion of the Nxnl1 gene in cones reduces the damage produced by oxidative stress. Silencing the expression of RdCVFL in cone-enriched culture reduces cell viability showing that RdCVFL is a cell-autonomous mechanism of protection.

Innovation: This novel mode of action is certainly relevant for the therapy of retinitis pigmentosa since the delivery into cones of the $r d 10$ mouse, a recessive model of the disease, rescues cones.

Conclusion: Our work highlights the duality of the $N x n / 1$ gene which protects the cones by two distinct mechanisms. 


\section{Introduction}

Cone photoreceptors have been shown to be particularly vulnerable to oxidative stress $(10,22,23,36,37)$. In that respect, it is of interest to notice that the nucleoredoxin-like one $(N x n / 1)$ gene, which encodes by alternative splicing rod-derived cone viability factor (RdCVF), also encodes RdCVFL that contains the thioredoxin fold as well as the catalytic CXXC domain $(4,26)$. Thioredoxins participate in redox homeostasis by reducing oxidized thiol groups in target proteins (28). Thioredoxins are oxidized during that reaction and must then be reduced by thioredoxin reductases that may be redox sensors of oxidative environment (38). Specifically, RdCVFL interacts with the microtubule associated protein TAU and prevents its oxidation in vitro. It also prevents its phosphorylation and aggregation in the retina $(10,15)$. RdCVFL protects rod photoreceptors against photo-oxidative damage (12) and reduces the oxidation of polyunsaturated fatty acids induced by photoreceptor degeneration in the $r d 10$ mouse (6). The rd10 mouse is a model of retinitis pigmentosa, the most common form of inherited retinal degenerations. We have shown that delivering RdCVFL to the retina under the control of a ubiquitous promoter does not prevent cone degeneration, unlike RdCVF (11). RdCVF production results from intron retention following transcription of the $N x n / 1$ gene. The phylogenic conservation of an in frame stop codon in RdCVF messenger produces a shorter protein, truncated within its thioredoxin motif (8). The 109 residues of RdCVF are identical to the $\mathrm{N}$ terminal region of the RdCVFL protein which is 217 amino acids long. RdCVF has no thioloxidoreductase activity even though it contains the thioredoxin catalytic site sequence CXXC (26). The effect of RdCVF on cones is mediated by its interaction with basigin-1 (BSG1), a splice variant of the $B s g$ gene expressed specifically by photoreceptors (1). The interaction of RdCVF with BSG1 at the surface of the cones stimulates glucose uptake by accelerating glucose transport by GLUT1. Cone rescue is the result of the production of metabolites by aerobic glycolysis that are probably used for cone outer segments renewal to sustain cone 
function. RdCVF is a promising therapeutic for retinitis pigmentosa, for which the degeneration of rods by a cell autonomous mechanism leads to night blindness, a mild condition, but is irremediably followed by the non-cell autonomous death of cones, which leads to loss of daylight vision $(7,27)$.

We envisioned that in healthy organisms, the active thioredoxin RdCVFL would be the sensor of luminance in rods participating, somehow in the production of a proper level of trophic factor RdCVF to cones adapted to light environment. Here, we challenged this view by inactivating the Nxnl1 specifically in cones. We show that cones expressed the thioredoxin RdCVFL that is involved in their protection against oxidative stress generated during aging. Our work supports further development of a novel double-therapeutic approach for retinitis pigmentosa. 


\section{Results}

\section{Specific disruption of $\mathbf{N x n l 1}$ gene in cones.}

To inactivate the $N x n / 1$ gene in cones, we crossed the $N x n / 1 c d / c d$ mouse line that carries two LoxP sites framing exon 1 of the gene (10) with the Cone-Cre mouse, a transgenic line that expresses Cre recombinase under the control of the $2.1 \mathrm{~kb}$ human OPN1L/MW (red and green opsins) promoter to obtain animals with the [Cone-Cre:Nxn/1cd/cd] genotype and their controls [Cone-Cre:Nxn/1+/+] (24). The recombination was validated and quantified using DNA extracted from sorted cells labeled with antibodies against mouse OPN1MW, the red/green opsins (Supplementary Figure 1). PCR primer pairs P1/P2 detected the wild-type (wt) alleles of the Nxn/1+/+ sorted cells and the pair P3/R4 the recombinated (rc) alleles of the Nxn/1-/- sorted cells (Figure 1A and 1B). Cells sorted from the [Cone-Cre: $N x n / 1 \mathrm{~cd} / \mathrm{cd}$ ] retina carry exclusively the rc allele at 554 base pair (bp) and cells sorted from the [ConeCre: $N x n / 1+/+]$ retina, carry the wt allele at $575 \mathrm{bp}$. The amplification of the Pde6b gene was used as an internal control. In order to quantify the recombination, we performed quantitative PCR analysis of the rc allele on DNA isolated from the whole retina using the primer pair P3/R4. $100 \%$ and $0 \%$ recombination correspond to the value obtained with $N \times n / 1-/-$ and $N x n / 1+/+$ retinas respectively (Figure $\mathbf{1 C}$ ). This scale shows that the recombination took place in about $2.5 \%$ of the [Cone-Cre: $N x n / 1 \mathrm{~cd} / \mathrm{cd}$ ], a value that corresponds approximately to the percentage of MW cones in the mouse retina. We also quantified $N x n / 1$ gene expression in the retina using RT-PCR. Using primers internal to the recombinated locus (P1/P2) we did not amplified the Nxnl1 messenger in the Nxn/1-/- retina, as expected. We could not differentiate Nxn/1 expression level in [Cone-Cre:Nxn/1cd/cd] retina from that of the [Cone-Cre:Nxn/1+/+] mouse showing that the recombination did not occur in the rods ( $97 \%$ of all photoreceptors) and is consequently specific to cones (Figure 1D). Similarly, using specific primer pairs, we observed similar expression of RdCVF and RdCVFL messengers in [Cone-Cre:Nxn/1cd/cd] and [Cone- 
Cre:Nxn/1cd/cd] retinas (Figure 1E and F). Using western blotting, we show that the expression of RdCVFL in the rods, the most numerous photoreceptors can be detected after recombination of $N x n / 1$ in the cones at 1 month of age (Figure 1G).

The function of cones was measured at 8 months of age using electroretinography (ERG). The traces show b-waves when tested by photopic ERG recording (Figure 2A). As previously reported, the amplitude of the photopic b-wave is reduced for Nxn/1-/- mice compared to their control, the Nxn/1+/+ mice (10) (Figure 2A and B). Surprisingly, while the bwave amplitude for the [Cone-Cre:Nxn/1+/+] is comparable to that of the $N x n / 1+/+$ mice, that of the [Cone-Cre:Nxn/1cd/cd] resembles that of the Nxn/1-/- mouse even though the cones (comprising only $3 \%$ of photoreceptors in the mouse retina) are surrounded by rods expressing the trophic factor RdCVF. We noticed that the trace of the b-wave differs between the $N \times n / 1+/+$ mice and the [Cone-Cre:Nxnl1+/+]. Since the only difference between the two strains is that cones of the [Cone-Cre:Nxn/1+/+] mice express the recombinase Cre under the control $2.1 \mathrm{~kb}$ human OPN1L/MW promoter, the difference might be linked to an unknown effect of the recombinase on ERG response but more likely a phenomenon of transcriptional interference between the locus control region (LCR) of the transgene with the endogenous expression of cone opsins resulting in a reduction of cone opsin expression revealed in the trace of the bwave $(21,34)$. This effect may be mediated by transcriptional squelching for transcription factors binding to the LCR $(16,17)$. The phenomenon is not strong enough to modify b-wave amplitude and does not interfere with our conclusions. Since cone-photopic ERG is a mixed ON-conebipolar and Muller cells response, we used flicker ERG that records specifically cone responses (Figure 2C). Here again, [Cone-Cre:Nxn/1cd/cd] flicker amplitude at $10 \mathrm{~Hz}$ and $20 \mathrm{~Hz}$ is reduced compared to [Cone-Cre:Nxn/1+/+] control mice (Figure 2D, E and Supplementary Figure 2A). We also look at the implicit time that scores cone photoreceptor response independently of the amount of light delivered to the retina (Supplementary Figure 2B). The 
implicit time is retarded by $12 \mathrm{~ms}(56$ to 68$)$ in the [Cone-Cre:Nxn/1cd/cd] mice compared to the [Cone-Cre:Nxn/1+/+] mice (Supplementary Figure 2C). We sacrificed the animals, labeled the cones with a mixture of OPN1MW and OPN1SW antibodies and measured the density of the cones over the entire surface of the retina $(1,9)$. The density of cones was found to be lower and similar for the $\mathrm{Nxn} / 1-/-$ and the [Cone-Cre:Nxnl1cd/cd] retinas compared to that of the [ConeCre:Nxn/1+/+] retinas (Figure $2 \mathbf{F}$ and $\mathbf{G}$ ). When we measured the density of cones at 4 months of age, we also found it lower for the [Cone-Cre: $N x n / 1 \mathrm{~cd} / \mathrm{cd}$ ] retinas compared to that of the [Cone-Cre:Nxn/1+/+] retinas (Supplementary Figure 2D). When we observed the flat-mounted retina at higher magnification, we observed that the labeling of opsins was distinct between the two genotypes in the ventro-nasal part of the retina (Supplementary Figure S2E). This is certainly a indication of a dimorphism of cones of the [Cone-Cre: $N x n / 1 \mathrm{~cd} / \mathrm{cd}$ ] mice that precedes their degeneration in this area.

\section{The cones are sensitive to oxidative stress even in the presence of rods.}

Since the cones in the [Cone-Cre:Nxn/1cd/cd] retinas are exposed to the trophic activity of RdCVF secreted by rods (1), their death cannot arise from a lack of trophic support. We housed one-month old animals in two different conditions. One of the groups was maintained in normoxic conditions $\left(21 \% \mathrm{O}_{2}\right)$, while the other group was maintained for one week in hyperoxia, in an atmosphere composed of $75 \%$ oxygen. At this age, photopic ERG responses of [ConeCre:Nxnl1cd/cd] and [Cone-Cre:Nxnl1+/+] control mice are more similar than traces from mice at 8 months of age in line with the progression of cone degeneration with age (Figure $3 \mathbf{A}$ ). Hyperoxia reduces photopic b-wave amplitudes for all genotypes but the amplitudes for the [Cone-Cre:Nxnl1+/+] mice under hyperoxia match those of the [Cone-Cre:Nxnl1cd/cd] mice under normoxia (Figure $\mathbf{3 A}$ and $\mathbf{B}$ ). Interestingly, the reduction in amplitude is similar for both the Nxn/1-/- and the [Cone-Cre:Nxn/1cd/cd] mice suggesting that the observed deficit is independent of RdCVF. When looking at a pure cone response using flicker ERG, the 
phenomenon was exacerbated (Figure 3C). Under normoxia, the three groups of animals tested have quite similar responses (Figure 3D). Under hyperoxia, the amplitude of the [ConeCre:Nxnl1+/+] flicker ERG is only marginally reduced by $\mathrm{O}_{2}$ corroborating previously published results (10). Using this assay, the contribution of RdCVF could be observed with a more dramatic reduction of the flicker amplitude at $10 \mathrm{~Hz}$ and $20 \mathrm{~Hz}$ (Figure 3C, 3D, Supplementary Figure $3 \mathbf{A}$ and $\mathbf{B}$ ) for the $N \times n / 1^{-/-}$compared to the [Cone-Cre: $\left.N x n / 1 c d / c d\right]$ mice. After sacrifice, the retinas were labeled with a mixture of OPN1MW and OPN1SW antibodies and cone density was measured. Here again, the loss of cone function under hypoxia paralleled a reduction in the number of cones in the [Cone-Cre:Nxn/1cd/cd] mice (Figure 3E and F). The similarity in cone density of the Nxn/1-/- and the [Cone-Cre:Nxn/1cd/cd] mice under hyperoxia, while they differ in cone flicker ERG amplitudes, may indicate that in the Nxn/1-/- mice without trophic support of RdCVF, cones loss their function before they degenerate. We examined the function and the survival of rods in the mice at 1 month of age in the presence or absence of oxidative stress. Scotopic ERG were recorded (Supplementary Figure 3C) and a-wave, characteristic of rod function was found unaffected under increasing flash intensity in any conditions (Supplementary Figure 3D). We found that the b-wave that results from more downstream signaling at the level of bipolar cells shows a trend toward lower amplitude for [ConeCre:Nxn/1cd/cd] mice as well as reduced amplitude in $75 \%$ oxygen (Supplementary Figure 3E). The thickness of the outer nuclear layer (ONL), composed of $97 \%$ rods was measured by optical coherence tomography in those animals (Supplementary Figure 3F). No differences could be observed between the two genotypes. Along the temporo-nasal retinal axis, the ONL was found slightly reduced in the [Cone-Cre:Nxn/1+/+] mice exposed to $75 \%$ oxygen on the temporal side (Supplementary Figure 3G). Along the dorso-ventral retinal axis, the ONL was found slightly reduced in the [Cone-Cre:Nxn/1+/+] mice exposed to $75 \%$ oxygen on the ventral side (Supplementary Figure 3G). Overall, these results show that the function and the survival of rods are not affected by deletion of Nxnl1 in $3 \%$ of photoreceptors, the cones. The 
contralateral eye of the mice were enucleated and fixed to perform immunohistochemistry. We observed, as previously reported, that the $N \times n / 1^{-/}$retina shows signs of oxidative damage using both anti-acrolein or 4-hydroxynonenal (HNE), two products of lipid peroxidation under oxidative stress (10) (Figure 4). Under hyperoxia, both [Cone-Cre:Nxn/1+/+] and [Cone-Cre:Nxn/1cd/cd] retinas were labeled with these antibodies, but the acrolein staining was more pronounced in the later group.

\section{The cones express RdCVFL, the thioredoxin protein product of the Nxn/1 gene.}

Since [Cone-Cre:Nxn/1cd/cd] mice have an age-dependant deficit in cone function and survival that can be accelerated by hypoxia, most likely the $N x n / 1$ gene is expressed by cones in wild-type (wt) retinas. We produced a reporter adeno-associated viral (AAV) vector expressing GFP under the control of the human OPN1L/MW promoter (39). We used an AAV variant called AAV2-7m8 that can target photoreceptors following intravitreal injection (11). On retinal explants of wt mice (BALB/c) injected with this vector, the fluorescence of GFP is colocalized with the expression of OPN1MW revealed in red by immunohistochemistry (Figure 5A). Individual GFP-positive cells were collected by micro-aspiration with patch-clamp glass electrodes. Current responses to voltage steps were recorded in some of these cells using the patch-clamp technique. Voltage steps ranged from $-100 \mathrm{mV}$ to $40 \mathrm{mV}$ (10 mV increments). Below $-60 \mathrm{mV}$, only weak linear inward currents were observed, and the cells typically exhibited a large outward current at potentials above $-40 \mathrm{mV}$, as expected for cone photoreceptors (Figure 5B and C) (32). After patch-clamp recordings the cones were used for single-cell RTPCR analysis. We used a negative (0) and a positive control that correspond to no CDNA and cDNA from wt retina (BALB/c) at post-natal day (PN) 40 respectively. The GFP cDNA shows that the RNA was collected from cones that do not express the rod photoreceptor marker, Rho (Figure 5D). We could amplify with specific primers the messenger of the thioredoxin RdCVFL but not that of the trophic factor RdCVF. For further validation, we repeated the analyses on 12 
independent GFP-positive cells that all gave the same result (Figure 5E). The cones, at least the MW-cones, express the thioredoxin RdCVFL exclusively.

\section{The thioredoxin RdCVFL protects cones against oxidative stress.}

To further establish the role of the thioredoxin RdCVFL in the observed deficit of the cones, the AAV2/8 virus, that drives expression of RdCVFL or RdCVF or GFP under a CMV promoter were employed. The subretinal injection of this virus serotype can transduce photoreceptors efficiently (29). We delivered either RdCVF or RdCVFL by subretinal injection of recombinant $\mathrm{AAV} 2 / 8$ vectors into the [Cone-Cre:Nxn/1cd/cd] mouse at postnatal days 20 (PN20) and housed the animals for five days, between PN35 and 40, in 75\% $\mathrm{O}_{2}$. The reduction of the photopic b-wave amplitude in uninjected mice shows that the cones of the [ConeCre:Nxn/1cd/cd] mice were affected by hyperoxia at PN40 (Figure 6A). The delivery of GFP or RdCVF had no effect on the ERG b-wave amplitude and on its shape, the later revealed by the contralateral uninjected eye (Figure 6B, C and D) in the [Cone-Cre: $N x n / 1 c d / c d]$ mice. In contrast, RdCVFL prevented the reduction of the amplitude by $48 \%$ [(126 - 103) / (151 - 103)] compared to [Cone-Cre:Nxn/1+/+] control mice. The flicker ERG was also recorded (Figure 6G). Using this readout, the results show a $46 \%$ [(110 - 78) / (147 - 78)] protection by RdCVFL (Figure 6E, F, Supplementary Figure 4A-C). The implicit time was shortened from $63 \mathrm{~ms}$ for animals treated with AAV-GFP to $60.5 \mathrm{~ms}$ for those treated with AAV-RdCVFL (Supplementary Figure 4D). The data are correlated (Supplementary Figure 4E). The animals were sacrificed and the cone density was quantified after labeling cones with peanut agglutinin (PNA), lectin from Arachis hypogaea. RdCVFL protected the cones by 63\% [(4.7 - 4.2) / (5.0 - 4.2)] (Figure $6 \mathrm{H}$ and $\mathrm{I})$.

We have previously reported that cones exclusively express the RdCVFL (and not the RdCVF) messenger RNA in cone-enriched culture from chicken embryos (15). Therefore, we 
silenced the expression of RdCVFL in the cell culture system by electroporating a miRNA plasmid (1). In order to validate the efficacy of the miRNA molecule, we cloned the open reading frame of chicken RdCVFL under a CMV promoter, 3' to Renilla reniformis luciferase. The plasmids were co-transfected in HEK293 cells. $48 \mathrm{~h}$ later, the luciferase activity showed that the miRNA is efficient (Figure 7A). The viability of the cells was scored after 7 days in vitro (DIV) by counting the number of cells transduced by the RFP reporter (Figure 7C). Specific silencing of endogenous RdCVFL reduces cell survival in a dose-dependent manner compared to a scrambled miRNA construct (Figure 7B).

The thioredoxin RdCVFL protects cones in a model of retinitis pigmentosa.

We have previously shown that in the $r d 1$ mouse, a model of recessive retinitis pigmentosa, the expression of Nxnl1 is rod-dependent (33). Nxn/1 expression by bipolar cells is lost following rod degeneration even if the bipolar cells are not affected in that model. We reasoned that the expression of RdCVFL by cones may also be lost following rod degeneration. We used a similar genetic model, the $r d 10$ mouse that carries a mutation in the same gene, Pde6b, but with slower kinetic of degeneration, thereby allowing functional evaluation (6). We produced novel AAV2/8 vectors for transgene delivery to mouse cones under the control of the human OPN1L/MW promoter. The $r d 10$ mice were subretinally injected at PN20 in one eye with AAV2/8 vectors and the function of the cones was recorded at PN40, after rods had degenerated. There was a net decrease in cone function in the rd10 mice compared to wt mice (Figure 8A and C). The transduction of cones with recombinant AAV vectors does not affect the shape of the photopic b-wave as seen in the contralateral uninjected eye (Figure 8B). Using this paradigm, RdCVF was not able to protect the cones, while RdCVFL protected cones by $36 \%[(102-55) /(187-55)]$. We also recorded the flicker ERG in those same animals (Figure 8D, E, F, and Supplementary Figure 5A). RdCVFL generated a $45 \%$ protection. We also noticed that the photopic and flicker ERG measurements are correlated for the $42 \mathrm{rd10}$ eyes 
included in the experiment (Supplementary Figure 5B). After sacrificing the mice, cone density measurements showed that RdCVFL prevented secondary cone death by $47 \%$ [(5.2 - 4.3) / (6.2 - 4.3)] (Figure 8G and $\mathbf{H}$ ). 


\section{Discussion}

We have demonstrated that the thioredoxin RdCVFL is normally expressed by cones as well as rods in the mouse retina. The role of this enzyme is to prevent the damage to cones observed in aged animals. Retinal damage is accelerated when the mice are exposed to a high level of oxygen. This cell-autonomous mechanism is very probably involved in the secondary cone degeneration in retinitis pigmentosa. In this disease, the loss of expression of the trophic factor RdCVF results in the lack of stimulation of aerobic glycolysis through its cell-surface receptor BSG1. The cones are deprived of glucose and their outer segments shorten because of the lack of production of proper glucose metabolites $(1,7,25)$. The reduction of the size of the cone outer segment is apparently not followed immediately by their death explaining why non-functional cones can be observed in the retinas of blind patients affected by retinitis pigmentosa (5). We have previously reported that the function of the cones in Nxn/1-/- mice deteriorates when the animals are exposed to hyperoxia (10). The deficit likely results from the absence of expression of RdCVFL in the cones rather than the lack of protection by the rods. What could be the mechanism explaining the vulnerability of cones to oxidation? For retinitis pigmentosa, the problem arises from the absence of regulation of choroidal circulation (35). The delivery of oxygen from the blood is maintained in the rod-less retina, even if its consumption is reduced by $97 \%$. Consequently, the retinal oxygen pressure reaches values that approximate hyperoxia. The cones and their outer segments composed of polyunsaturated fatty acids are damaged by reactive oxygen species produced from leakage of the respiratory chain. In the absence of $N x n / 1$, the retina shows signs of lipid peroxidation (10). After specific recombination of $N x n / 1$ gene in the cones, the retina also displayed signs of oxidative damage (Figure 4). The viability of the cones of the cone-enriched cultures depends on the endogenous protection by the thioredoxin RdCVFL. In that system, we showed that the survival by ectopic RdCVF is not dependent on redox homeostasis but on aerobic glycolysis (1). Nevertheless, in the retina, the 
thiol-oxidoreductase activity of RdCVFL depends on its cycling between a reduced and an oxidized status as any other thioredoxin. Once oxidized, RdCVFL must be reduced by thioredoxin reductases (20). The co-factor of thioredoxin reductases, NADPH, is produced mostly from glucose through the pentose phosphate pathway (PPP) (Figure 9). In that respect, in cones, RdCVF likely acts upstream of RdCVFL by providing reducing power. Many of the glycolytic enzymes are negatively regulated by oxidation of thiol groups at cysteine residues, like glyceraldehydes-3-phosphate dehydrogenase and pyruvate kinase (PKM) $(2,19)$. Oxidation of PKM at cysteine 358 (C358) stops the production of pyruvate and results in the accumulation of phosphoenol pyruvate (PEP). PEP, an allosteric inhibitor of triose phosphate isomerase, interrupts glycolysis which produces an elevation of the intracellular concentration of glucose-6phosphate (G6P). G6P, produced by the action of hexokinases on glucose entry to the cell via glucose transporters, is diverted to produce ribulose-5-phosphate (R5P) in PPP, enzymatic steps catalyzed by glucose-6-phosphate dehydrogenase (G6PDH) and 6-phosphogluconate dehydrogenase (PGD). Both G6PDH and PGD use NADP+ as a co-factor and that is reduced to NADPH during the reaction. NADPH is used by glutathione reductase to reduce glutathione and by thioredoxin reductases to reduce thioredoxins. Reduced RdCVFL could reestablish aerobic glycolysis in cones after oxidative stress and we have identified a potential interaction between RdCVFL and PKM (15). One could speculate that in patients suffering from retinitis pigmentosa after the loss of expression of RdCVF produced by rods, cones become non-functional and die within several years as a result of the loss RdCVFL expression and subsequent oxidative damage in cones. We have previously observed that the expression of $N x n / 1$ in cells neighboring the rods depends on the presence of rods (33). Thus, a therapy aimed at preventing secondary cone degeneration should be pursued using both RdCVF and RdCVFL. While RdCVF could be delivered to cones by any surrounding cells, excluding dying rods, RdCVFL would have to be transduced to the cone themselves to exert the cell-autonomous protection observed here. This might be achieved using ubiquitous or cone-restricted promoters. 
Regarding the evolution of RdCVF signaling, it is quite reasonable to propose that the thioredoxin activity precedes the trophic activity of RdCVF, the latter resulting from an alternative splicing event that produces a truncated thioredoxin (18). Cones evolved earlier than rods, the latter appeared during evolution at a period when a reduction in energy consumption favored cephalization (13). The alternative splicing event at the origin of the RdCVF metabolite signaling should be anterior to the diversification of the nucleoredoxin-like family by duplication of an ancestral $N x n l$ gene into $N x n / 1$ and $N x n / 2(6,8)$. Interestingly, the lamprey, a jaw-less fish, harbors two types of photoreceptors with cone morphology. One of them is possibly a rod in regard to its spectral sensitivity (31). Prior to its role in rod-cone interactions, the ancestral $N x n l$ gene may have been involved in redox homeostasis and possibly contributed to glycolysis. 


\section{Innovation}

Retinitis pigmentosa is a blinding disease characterized by the sequential loss of rod photoreceptors followed by the cones. The nucleoredoxin-like $1(N x n / 1)$ gene encodes by alternative splicing for a trophic factor, rod-derived cone viability factor (RdCVF) that is secreted by rods and protects cones by stimulating aerobic glycolysis. We show that the other product of this gene, the thioredoxin protein RdCVFL, is expressed by cones where it exerts protection against oxidative stress. This suggests that the protection of cone vision in retinitis pigmentosa should benefit from a double therapeutic approach where both products of the Nxnl1 gene are administrated. 


\section{Material and methods}

\section{Animals.}

The Nxn/1-/- mice on BALB/c background were generated previously (10). The BALB/C $(\mathrm{N} x n / 1+/+)$ mice were used as wt mice. The $N x n / 1 \mathrm{~cd} / \mathrm{cd}$ mice on BALB/c background contain loxP sites frame exon 1 of the Nxn/1 gene, and the Cone-Cre mice with Cre recombinase directed by the human red/green pigment (HRGP) gene were kindly provided by Yun Zheng Le (24). Cone-Cre mice were crossed with $\mathrm{Nxn} / 1+/+$ mice more than 10 generations to reach BALB/c background. [Cone-Cre:Nxn/1+/+] and [Cone-Cre:Nxn/1cd/cd] mice were obtained by crossing Nxn/1cd/cd mice with Cone-Cre mice. The $r d 10$ and C57BL/6J mice were purchased from Jackson laboratory. All mice were housed under the light/dark $(12 / 12 \mathrm{~h})$ cycle, $23-25^{\circ} \mathrm{C}$ room temperature and offered ad libitum access to food and water. The project was approved by French Ethics Committee ( $\mathrm{N}^{\circ} \mathrm{A}-75-1863$; OGM $\left.\mathrm{n}^{\circ} 5080 \mathrm{CA}-\mathrm{II}\right)$. And all experiments were performed in accordance with the European Community Council Directives of September 22, 2010 (2010/63/UE).

\section{Retinal cells preparation for FACS cone sorting.}

Mouse eyes were enucleated, their retina dissected. Retinas ( 2 retinas/tube) were dissociated in $450 \mu \mathrm{l} \mathrm{Hank's} \mathrm{balanced} \mathrm{salt} \mathrm{solution} \mathrm{(HBSS,} \mathrm{no} \mathrm{calcium)} \mathrm{with} 50 \mu \mathrm{g}$ trypsin for $5 \mathrm{~min}$ at $37^{\circ} \mathrm{C}$. To stop the reaction, the cells were mixed well with $1,000 \mu \mathrm{g}$ trypsin inhibitor in $450 \mu \mathrm{l} \mathrm{HBSS}$ (with calcium) and $50 \mu \mathrm{g}$ Dnase I by using a pipette and centrifuge $3 \mathrm{~min}$ at $2,000 \mathrm{~g}$ to keep the dissociated cells. The cell pellet was resuspended in $500 \mu \mathrm{l}$ phosphate buffer saline (PBS), and fixed for 30 min in $2 \%$ paraformaldehyde. The fixed cells were then washed with PBS and blocked in PBS containing $0.4 \%$ bovine serum albumin (BSA), $1 \%$ fetal bovine serum (FBS) and 
$1 \mathrm{mg} / \mathrm{ml}$ saponin to proceed for immunolabeling with rabbit anti-OPN1MW antibodies (1/40, Millipore AB5405) or negative control rabbit anti-lgG (1/40), and then secondary antibodies: Rphycoerythrin (1/50, Life technologies). The labeled cells were washed and maintained in $500 \mu \mathrm{l}$ PBS for FACS cone sorting.

\section{Analysis of homologous recombination by PCR.}

Genomic DNA from FACS-sorted cones was extracted with DNeasy Blood \& Tissue Kit

(Qiagen, 69504). PCR analysis were carried out using primers P1 (5'TACCTTGAGTGACACACC-3') / P2 (5'-CCCTCCCTGGTCTTCCCTGGGG-3') for wt allele, and $\begin{array}{lllll}\text { primers } & \text { P3 (5'-TCCACACACAGCACATCATACG-3') } & \text { / } & \text { R4 } & \text { (5'- }\end{array}$ TGTGATGGAAGAGGGATGGAAC-3') for recombined allele.

\section{Quantitative-PCR analysis.}

Total RNAs of mouse retina were extracted with kit (RNeasy mini kit, Qiagen). The RNA was reverse-transcribed with SuperScript ${ }^{\circledR}$ II reverse transcriptase kit (Life technologies). Samples have been analyzed by quantitative PCR using power SYBR green PCR master mix (Life technologies) in a 7500 standard real-time PCR system (Applied biosystems) with primers P1/P2, or P3/R4, RdCVF (5'-CTACAGAGGAGCAACAGGAC-3'; 3'TGCACAAGTAGTACCAGGAC-5') or RdCVFL (5'-GCAACAGGACCTCTTCCTCA-3'; 3'CCAGACGCTGGATCTCCTC-5').

\section{Western-blotting.}


Western blotting on mouse neural retina was performed as previously described using rabbit polyclonal antibodies RdCVF-N (26).

\section{Electroretinograms.}

Mice were anesthetized intraperitoneally with a mixture of ketamine $(80 \mathrm{mg} / \mathrm{kg})$ and xylazine (10 $\mathrm{mg} / \mathrm{kg}$ ) diluted in saline. Their pupils were dilated by topical application of $0.5 \%$ mydriaticum. Body temperature was maintained at $37^{\circ} \mathrm{C}$ with a circulating hot water heating pad. The electrical signal was recorded using a pair of electrodes constructed in our platform adapted for use on mice. A gold loop electrode was placed on the center of the corneal surface and maintained with ocrygel (TVM) to ensure good electrical contact. Two stainless steel reference electrodes were inserted subcutaneously in each cheek of the mouse to normalize signal output, and the ground electrode was inserted subcutaneously in the back of the mouse. Recordings were made from both eyes simultaneously. Recording measurements: The light stimulus was provided by a $150 \mathrm{~W}$ xenon lamp in a Ganzfeld stimulator (Multiliner vision, Jaeger toennies, Germany). Responses were amplified and filtered (1 Hz-low and $300 \mathrm{~Hz}$-high cut off filters) with a 1 channel DC-/AC-amplifier. To isolate cone responses in photopic, a 5 min light saturation at $10 \mathrm{cds} / \mathrm{m}^{2}$ was used to desensitize the rods. The cone photopic ERGs shown represents the average of 10 responses from 10 consecutive flashes at $10 \mathrm{cds} / \mathrm{m}^{2}$ intensity. The flicker ERG was also used to isolate cone responses at flash frequencies of 10 , and $20 \mathrm{~Hz}$ at 3 $\mathrm{cds} / \mathrm{m}^{2}$ intensity. Data acquisition: The photopic b-wave amplitudes were measured from the base-line to the peak. For scotopic recordings, single flash recordings were obtained at light intensities of $3 \times 10^{-3}, 3 \times 10^{-2}, 3 \times 10^{-1}, 3$ and $10 \mathrm{cds} / \mathrm{m}^{2}$ using a sampling frequency of $5 \mathrm{kHz}$, a flash duration of $4 \mathrm{~ms}$, and a frequency stimulus of $0.5 \mathrm{~Hz}$. Data were recorded from $50 \mathrm{~ms}$ before stimulus onset to $450 \mathrm{~ms}$ post-stimulus. Data acquisition: The scotopic a- and b-wave amplitudes were measured from the base-line to the peak. 


\section{Optical coherence tomography (OCT).}

Mice aged of 1 month were anesthetized and pupils were dilated. Eye dehydration was prevented by regular instillation of sodium chloride drops. OCT images were recorded on the right eye using a spectral domain ophthalmic imaging system (Spectral domain Optical coherence tomography, OCT, Bioptigen 840 nm HHP; Bioptigen; North Carolina USA). We performed rectangular scans consisting of a $2 \mathrm{~mm}$ by $2 \mathrm{~mm}$ perimeter with 1000 A-scans per Bscan with a total B-scan amount of 100 . Scans were obtained while centered on the optic nerve. OCT scans were exported and analyzed using ImageJ. Outer nuclear layer (ONL) thickness was measured every $100 \mu \mathrm{m}$, ventral-dorsal and temporal-nassal, starting $100 \mu \mathrm{m}$ from the center (optic nerve).

\section{Hyperoxia treatment.}

Mice were maintained in a $75 \%$ oxygen chamber for seven days with free access to food and water (BioSpherix, NY 13083).

\section{Immunohistochemistry.}

The eyes were enucleated quickly and immerged in 4\% paraformaldehyde in PBS overnight at $4^{\circ} \mathrm{C}$. They were incubated successively in $10 \%$ and $20 \%$ sucrose at $4^{\circ} \mathrm{C}$ and then embedded in optimal cutting temperature compound (Sakura finetek, Gentaur, Belgium). Frozen sections of $10 \mu \mathrm{m}$ thickness were obtained for immunohistochemistry. The sections were first pre-incubated $2 \times 30$ min in blocking buffer $5 \%$ BSA and tween-20 $0.1 \%$ in PBS). They were then processed to overnight incubation at $4{ }^{\circ} \mathrm{C}$ in 4-Hydroxynonenal (HNE) (Calbiochem, 1/300) or acrolein (Cosmo bio, Tokyo, Japan, 1/150) antibodies. After two washes of $30 \mathrm{~min}$, the sections were 
incubated $2 \mathrm{~h}$ at room temperature with goat anti-mouse IgG conjugated to alexa $594(1 / 500)$, lectin PNA conjugated to alexa 488 (1/100, Life technologies) and the nuclear marker DAPI (Sigma, 1/1000). The sections were washed 3 times with PBS before ultimately mounted with fluoromount-G (SouthernBiotech). Images have been obtained with a confocal microscope (Olympus FV1000) at $63 x$ objective. Stacks of consecutive images taken at $500 \mathrm{~nm}$ intervals were acquired sequentially with lasers: diode $559 \mathrm{~nm}$, argon $488 \mathrm{~nm}$ and diode $405 \mathrm{~nm}$ and Z projections of serial sections were reconstructed in the software (FV-10-ASW-4.2) coupled to confocal.

\section{Cone density measurement.}

Cone counting was performed after ERG recording. The retinas were carefully dissected in prewarmed $\left(37^{\circ} \mathrm{C}\right)$ PBS (Gibco, Life technologies) and transferred to $4 \%$ paraformaldehyde overnight at $4^{\circ} \mathrm{C}$. The retinas were prepared for immunostaining as described (9). After $1 \mathrm{~h} 30$ min saturation in PBS containing $1 \%$ BSA, $0.1 \%$ tween and $10 \%$ normal goat serum (NGS), the retinas were incubated with a mixture of OPN1SW [1/400, (14)] and OPN1MW antibodies $\left(1 / 200\right.$, Millipore) in the blocking buffer overnight at $4^{\circ} \mathrm{C}$. They were washed $3 \times 15$ min in PBS containing $0.1 \%$ tween-20 and $1 \%$ BSA $\left(\mathrm{PBS}^{*}\right)$ for goat anti-rabbit $\lg \mathrm{G}$ conjugated to alexa 488 $(1 / 400)$ incubation for $2 \mathrm{~h}$ at room temperature. Then they were re-washed $3 \times 15 \mathrm{~min}$ in PBS*, counterstained by $3 \mathrm{~h}$ incubation in lectin PNA conjugated to alexa 488 (1/100) (3) in the blocking buffer. After final washing in PBS, the retinas were whole-mounted photoreceptor sideup in fluoromount-G. Cone density was analyzed in an automated system developed (econome) as described previously (9). Row data are available on request.

\section{Single cell recording and harvest.}


The AAV2-7m8 OPN1L/MW-GFP injected eyes were enucleated; their retinas were dissected and dissociated 5 min by papain ( 1 unit/retina). Dissociated cells were then resuspended in RINGER solution (125 mM NaCl, $3.6 \mathrm{mM} \mathrm{KCl,} 1.18 \mathrm{mM} \mathrm{MgCl}$, $22.6 \mathrm{mM} \mathrm{NaHCO}, 0.02 \mathrm{mM}$ $\mathrm{NaH}_{2} \mathrm{PO}_{4}, 0.028 \mathrm{mM} \mathrm{Na} \mathrm{HPO}_{4}, 1.2 \mathrm{mM} \mathrm{Na} \mathrm{SO}_{4}, 10 \mathrm{mM}$ Glucose, $0.54 \mathrm{mM}$ EDTA) for whole cell recording. Recording pipettes were pulled from borosilicate glass capillaries (BF100-50-10, Sutter Instruments) with a next generation micropipette puller (P-1000; Sutter Instruments). Single GFP-expressed cell was voltage-clamped to measure membrane currents. The recorded cell was collected in $5 \mu$ PBS in a $1.5 \mathrm{ml}$ Eppendorf tube.

\section{Single cell RT-PCR analysis.}

Single cell cDNA was amplified according to the protocol described in Morris et al. (30).

\section{AAV injection.}

Mice were anesthetized with isoflurane (IsoFlo, France) inhalation. They were first placed in a container connected with the vaporizer (Tec 7, Anestéo, France). Anesthesia was induced by inhalation of $5 \%$ vaporized isoflurane within $30 \mathrm{~s}$. During the injection, anesthesia was maintained by mask inhalation of $2 \%$ vaporized isoflurane (debit $2 \mathrm{l} / \mathrm{min}$ ). For intravitreal injection, the micro-syringe (10 $\mu \mathrm{l}$, Hamilton, Reno, NY) was gently inserted into the vitreous cavity. A total of $2 \times 10^{10} \mathrm{vg}$ (viral genome) AAV in a $1 \mu \mathrm{l}$ volume was subsequently injected into the vitreous cavity. For sub-retinal injection, a small hole was performed with a 10-0 needle in the superior sclera. The micro-syringe was then gently inserted into the sub-retinal space, and 1 $\mu \mathrm{l}$ of $\operatorname{AAV}\left(2 \times 10^{13} \mathrm{vg} / \mathrm{ml}\right.$ titer $)$ was injected. The local retinal detachment was checked to confirm the success introduction of the virus. 


\section{miRNA construction and electroporation in cone-enriched culture.}

The miRNA sequence used was designed with BLOCK-iT Pol II miR RNAi (Life technologies). The miRdCVFL (5'-AGTGTGCCAGGAGAAGCATCA-3'), targets the sequence at positions 512532 from chicken RdCVFL mRNA 5'-end (XM_423688). This miRdCVFL or its scrambled (5'GAGTACAAGTCGCGTACGAAG-3') was cloned into pcDNA6.2 GW/EmGFP-miR (Life technologies) vectors. At the same time, chicken RdCVFL open reading frame (ORF) sequence (624 bp) was cloned under the control of a CMV promoter in pRL-CMV (Promega), 3' to the Renilla reniformis luciferase to obtain $\mathrm{pRL}-\mathrm{CMV}-\mathrm{RdCVFL-Ren.} \mathrm{This} \mathrm{plasmid} \mathrm{was} \mathrm{co-transfected}$ with miRdCVFL or scramble plasmid in HEK293 cells using Lipofectamine 2000 in OptiMEM medium (Life technologies). $48 \mathrm{~h}$ later, Renilla luciferase activity was measured from cell extracts using dual-luciferase reporter assay system (Promega) to validate the efficacy of the miRdCVFL molecule. For live-dead assay, the delivery of the miRdCVFL from pcDNA6.2 GW/EmGFP-miRdCVFL construct and the RFP-reporter from pCAG-G-RFP plasmid was performed by co-electroporation into cone-enriched-chicken cultures as previously described (1). The RFP-reporter was served as a marker of the transduced cells. Seven days after electroporation, the RFP-expressing living cells were counted on an automated microscopy system.

\section{Statistics.}

All measurements were made using blind protocols. Statistical significance was analyzed with Student's t-test to compare the means of 2 groups. When 3 or more groups were compared, one- or two-way-ANOVA Tukey's test has been applied (Prism 6; GraphPad software). In the figures, different levels of significance are indicated as: ${ }^{*}$ for $p<0.05 ;{ }^{* *}$ for $p<0.01$; ${ }^{* * *}$ for $p<0.001 ;{ }^{* * * *}$ for $p<0.0001$. 


\section{Acknowledgments}

We thank Yun Zheng Le, Hanen Khabou and Manu simonutti, Julie Degardin and Quenol Cesar of the phenotyping platform of the Institut de la Vision for biological materials and help. This work was supported by UPMC, Inserm, CNRS, the European commission, FFB and ANR. 


\section{List of abbreviations}

AAV: Adeno-associated viral vector

ANR: Agence nationale de la recherche

bp: base pair

BSA: bovine serum albumin

Bsg: Basigin gene

BSG1: Basigin-1 protein

CMV: Cytomegalovirus

CNRS: Centre national de la recherche scientifique

DNA: Deoxyribonucleic acid

Dnase I: Deoxyribonuclease I

ERG: Electroretinography

FACS: Fluorescence-activated cell sorting

FFB: Foundation fighting blindness

FBS: Fetal Bovine Serum

GFP: Green fluorescent protein

G6P: Glucose-6-phosphate

G6PDH: Glucose-6-phosphate dehydrogenase

GLUT1: glucose transporter SLC2A1

HBSS: Hank's balanced salt solution

HNE: 4-Hydroxynonenal

Ig: Immunoglobulin

Inserm: Institut national de la santé et de la recherche médicale

miRNA: micro-ribonucleic acid

NADPH: Nicotinamide adenine dinucleotide phosphate, reduced form

NADP+: Nicotinamide adenine dinucleotide phosphate, oxidized form 
NGS: Normal Goat Serum

Nxn11: Nucleoredoxin-like-1 gene

Nxn/2: Nucleoredoxin-like-1 gene

OCT: Optical coherence tomography

OPN1L/MW: Long (red) and medium (green) wavelength opsins

OPN1MW: Medium (green) wavelength opsin

OPN1SW: Short (blue) wavelength opsin

ONL: Outer nuclear layer

PGD: 6-phosphogluconate dehydrogenase

PCR: Polymerase chain reaction

PEP: Phosphoenol pyruvate

PBS: Phosphate buffer saline

PKM: Pyruvate kinase isoform $M$

PN: Post-natal day

PNA: Peanut agglutinin

PPP: Pentose phosphate pathway

R5P: Ribulose-5-phosphate

rc: recombinated allele

RdCVFL: Rod-derived cone viability factor long

RdCVF: Rod-derived cone viability factor

RFP: Red fluorescent protein

RNA: Ribonucleic acid

TAU: Microtubule-associated protein $\tau$

UPMC: Université Pierre et Marie Curie

vg: viral genome

wt: wild-type 


\section{References}

1. Ait-Ali N, Fridlich R, Millet-Puel G, Clerin E, Delalande F, Jaillard C, Blond F, Perrocheau L, Reichman S, Byrne LC, Olivier-Bandini A, Bellalou J, Moyse E, Bouillaud F, Nicol X, Dalkara D, van Dorsselaer A, Sahel JA, Leveillard T. Rod-derived cone viability factor promotes cone survival by stimulating aerobic glycolysis. Cell 161: 817-32, 2015.

2. Anastasiou D, Poulogiannis G, Asara JM, Boxer MB, Jiang JK, Shen M, Bellinger G, Sasaki AT, Locasale JW, Auld DS, Thomas CJ, Vander Heiden MG, Cantley LC. Inhibition of pyruvate kinase M2 by reactive oxygen species contributes to cellular antioxidant responses. Science 334: 1278-83, 2011.

3. Blanks JC, Johnson LV. Selective lectin binding of the developing mouse retina. $J$ Comp Neurol 221: 31-41, 1983.

4. Brennan LA, Lee W, Kantorow M. TXNL6 is a novel oxidative stress-induced reducing system for methionine sulfoxide reductase a repair of alpha-crystallin and cytochrome $\mathrm{C}$ in the eye lens. PLoS One 5: e15421, 2010.

5. Busskamp V, Duebel J, Balya D, Fradot M, Viney TJ, Siegert S, Groner AC, Cabuy E, Forster V, Seeliger M, Biel M, Humphries $P$, Paques M, Mohand-Said S, Trono D, Deisseroth K, Sahel JA, Picaud S, Roska B. Genetic reactivation of cone photoreceptors restores visual responses in retinitis pigmentosa. Science 329: 413-7, 2010.

6. Byrne LC, Dalkara D, Luna G, Fisher SK, Clerin E, Sahel JA, Leveillard T, Flannery JG. Viral-mediated RdCVF and RdCVFL expression protects cone and rod photoreceptors in retinal degeneration. J Clin Invest 125: 105-16, 2015.

7. $\quad$ Cepko C, Punzo C. Cell metabolism: Sugar for sight. Nature 522: 428-9, 2015.

8. Chalmel F, Leveillard T, Jaillard C, Lardenois A, Berdugo N, Morel E, Koehl P, Lambrou G, Holmgren A, Sahel JA, Poch O. Rod-derived Cone Viability Factor-2 is a novel bifunctional-thioredoxin-like protein with therapeutic potential. BMC Mol Biol 8: 74, 2007.

9. Clerin E, Wicker N, Mohand-Said S, Poch O, Sahel JA, Leveillard T. e-conome: an automated tissue counting platform of cone photoreceptors for rodent models of retinitis pigmentosa. BMC Ophthalmol 11: 38, 2011.

10. Cronin T, Raffelsberger W, Lee-Rivera I, Jaillard C, Niepon ML, Kinzel B, Clerin E, Petrosian A, Picaud S, Poch O, Sahel JA, Leveillard T. The disruption of the rod-derived cone viability gene leads to photoreceptor dysfunction and susceptibility to oxidative stress. Cell Death Differ 17: 1199-210, 2010.

11. Dalkara D, Byrne LC, Klimczak RR, Visel M, Yin L, Merigan WH, Flannery JG, Schaffer DV. In vivo-directed evolution of a new adeno-associated virus for therapeutic outer retinal gene delivery from the vitreous. Sci Transl Med 5: 189ra76, 2013.

12. Elachouri G, Lee-Rivera I, Clerin E, Argentini M, Fridlich R, Blond F, Ferracane V, Yang Y, Raffelsberger W, Wan J, Bennett J, Sahel JA, Zack DJ, Leveillard T. Thioredoxin rodderived cone viability factor protects against photooxidative retinal damage. Free Radic Biol Med 81C: 22-29, 2015.

13. Fain GL, Hardie R, Laughlin SB. Phototransduction and the evolution of photoreceptors. Curr Biol 20: R114-24, 2010.

14. Fintz AC, Audo I, Hicks D, Mohand-Said S, Leveillard T, Sahel J. Partial characterization of retina-derived cone neuroprotection in two culture models of photoreceptor degeneration. Invest Ophthalmol Vis Sci 44: 818-25, 2003.

15. Fridlich R, Delalande F, Jaillard C, Lu J, Poidevin L, Cronin T, Perrocheau L, Millet-Puel G, Niepon ML, Poch O, Holmgren A, Van Dorsselaer A, Sahel JA, Leveillard T. The thioredoxin-like protein rod-derived cone viability factor (RdCVFL) interacts with TAU and inhibits its phosphorylation in the retina. Mol Cell Proteomics 8: 1206-18, 2009. 
16. Gilbert DM, Heery DM, Losson R, Chambon $\mathrm{P}$, Lemoine $\mathrm{Y}$. Estradiol-inducible squelching and cell growth arrest by a chimeric VP16-estrogen receptor expressed in Saccharomyces cerevisiae: suppression by an allele of PDR1. Mol Cell Biol 13: 462-72, 1993.

17. Gill G, Ptashne M. Negative effect of the transcriptional activator GAL4. Nature 334: 721-4, 1988.

18. Hertel KJ. Spliceosomal pre-mRNA splicing methods and protocols New York u.a.: Humana Press; 2014. XI, 427 S. p.

19. Hildebrandt T, Knuesting J, Berndt C, Morgan B, Scheibe R. Cytosolic thiol switches regulating basic cellular functions: GAPDH as an information hub? Biol Chem 396: 52337, 2015.

20. Holmgren A. Thioredoxin. Annu Rev Biochem 54: 237-71, 1985.

21. Hu X, Eszterhas S, Pallazzi N, Bouhassira EE, Fields J, Tanabe O, Gerber SA, Bulger $M$, Engel JD, Groudine M, Fiering S. Transcriptional interference among the murine beta-like globin genes. Blood 109: 2210-6, 2007.

22. Komeima K, Rogers BS, Lu L, Campochiaro PA. Antioxidants reduce cone cell death in a model of retinitis pigmentosa. Proc Natl Acad Sci U S A 103: 11300-5, 2006.

23. Komeima K, Usui S, Shen J, Rogers BS, Campochiaro PA. Blockade of neuronal nitric oxide synthase reduces cone cell death in a model of retinitis pigmentosa. Free Radic Biol Med 45: 905-12, 2008.

24. Le YZ, Ash JD, Al-Ubaidi MR, Chen Y, Ma JX, Anderson RE. Targeted expression of Cre recombinase to cone photoreceptors in transgenic mice. Mol Vis 10: 1011-8, 2004.

25. Leveillard T. Cancer metabolism of cone photoreceptors. Oncotarget 6: 32285-6, 2015.

26. Leveillard T, Mohand-Said S, Lorentz O, Hicks D, Fintz AC, Clerin E, Simonutti M, Forster V, Cavusoglu N, Chalmel F, Dolle P, Poch O, Lambrou G, Sahel JA. Identification and characterization of rod-derived cone viability factor. Nat Genet 36: 7559, 2004.

27. Leveillard T, Sahel JA. Rod-derived cone viability factor for treating blinding diseases: from clinic to redox signaling. Sci Transl Med 2: 26ps16, 2010.

28. Lu J, Holmgren A. The thioredoxin antioxidant system. Free Radic Biol Med 66: 75-87, 2014.

29. Manfredi A, Marrocco E, Puppo A, Cesi G, Sommella A, Della Corte M, Rossi S, Giunti M, Craft CM, Bacci ML, Simonelli F, Surace EM, Auricchio A. Combined rod and cone transduction by adeno-associated virus 2/8. Hum Gene Ther 24: 982-92, 2013.

30. Morris J, Singh JM, Eberwine JH. Transcriptome analysis of single cells. J Vis Exp, 2011.

31. Morshedian A, Fain GL. Single-Photon Sensitivity of Lamprey Rods with Cone-like Outer Segments. Current Biology 25: 484-487, 2015.

32. Pattnaik B, Jellali A, Sahel J, Dreyfus H, Picaud S. GABAC receptors are localized with microtubule-associated protein $1 \mathrm{~B}$ in mammalian cone photoreceptors. J Neurosci 20: 6789-96, 2000.

33. Reichman S, Kalathur RK, Lambard S, Ait-Ali N, Yang Y, Lardenois A, Ripp R, Poch O, Zack DJ, Sahel JA, Leveillard T. The homeobox gene CHX10/VSX2 regulates RdCVF promoter activity in the inner retina. Hum Mol Genet 19: 250-61, 2010.

34. Smallwood PM, Wang Y, Nathans J. Role of a locus control region in the mutually exclusive expression of human red and green cone pigment genes. Proc Natl Acad Sci U S A 99: 1008-11, 2002.

35. Stone J, Maslim J, Valter-Kocsi K, Mervin K, Bowers F, Chu Y, Barnett N, Provis J, Lewis G, Fisher SK, Bisti S, Gargini C, Cervetto L, Merin S, Peer J. Mechanisms of photoreceptor death and survival in mammalian retina. Prog Retin Eye Res 18: 689-735, 1999. 
36. Usui S, Komeima K, Lee SY, Jo YJ, Ueno S, Rogers BS, Wu Z, Shen J, Lu L, Oveson BC, Rabinovitch PS, Campochiaro PA. Increased expression of catalase and superoxide dismutase 2 reduces cone cell death in retinitis pigmentosa. Mol Ther 17: 778-86, 2009.

37. Xiong W, MacColl Garfinkel AE, Li Y, Benowitz LI, Cepko CL. NRF2 promotes neuronal survival in neurodegeneration and acute nerve damage. J Clin Invest 125: 1433-45, 2015.

38. Xu J, Eriksson SE, Cebula M, Sandalova T, Hedstrom E, Pader I, Cheng Q, Myers CR, Antholine WE, Nagy P, Hellman U, Selivanova G, Lindqvist Y, Arner ES. The conserved Trp114 residue of thioredoxin reductase 1 has a redox sensor-like function triggering oligomerization and crosslinking upon oxidative stress related to cell death. Cell Death Dis 6: e1616, 2015.

39. Zack DJ, Bennett J, Wang Y, Davenport C, Klaunberg B, Gearhart J, Nathans J. Unusual topography of bovine rhodopsin promoter-lacZ fusion gene expression in transgenic mouse retinas. Neuron 6: 187-99, 1991.

\section{Figure legends.}

\section{Figure 1.}

Homologous recombination of the Nxn/1 gene in the cones of the [Cone-Cre:Nxn/1cd/cd] mice. Position of P1/P2 and P3/R4 primers within the nxn/1 gene (A). PCR analysis in FACS-sorted cones of Nxnl1+/+, Nxnl1-/-, [Cone-Cre:Nxn/1+/+] and [Cone-Cre:Nxnl1cd/cd] mice, negative control (0). Top row: the amplification of exon1 (P1, P2). Middle row: the amplification by primers (P3, R4) framing exon1. The rod-specific-phosphodiesterase-6b gene $(P d e 6 b)$ is an internal control (B). Real-time PCR quantification with primers $(P 3, R 4)$ in the DNA extracted from mouse retinas ( $n=3$ mice). Cre-mediated recombination took place in about $2.5 \%$ of the retinal cells in [Cone-Cre:Nxn/1cd/cd] retina as compared to Nxn/1-/- mice ( $\mathrm{n}=3$ mice) (C). Realtime PCR quantification of Nxnl1 gene in the Nxnl1+/+, Nxn/1-/-, [Cone-Cre:Nxnl1+/+] and [Cone-Cre:Nxn/1cd/cd] mice retina with P1/P2 ( $n=3$ mice) (D). Quantification of RdCVF cDNA expression ( $n=3$ mice) (E). Quantification of RdCVFL cDNA expression $(n=5)(F)$. Western blotting analysis of retinal extracts of mice aged 1 month using anti-RdCVF-N antibodies. ACTB: cytoplasmic actin (G). a.u.: arbitrary unit. Error bars correspond to standard of the mean (SEM). 


\section{Figure 2.}

Cone function and viability of cones of the [Cone-Cre: $\mathrm{Nxn} / 1 \mathrm{~cd} / \mathrm{cd}$ ] mice are reduced at 8 months of age. Representative photopic ERG recordings (A). Amplitudes of photopic ERGs ( $n=10$ mice) (B). Representative $10 \mathrm{~Hz}$ flicker ERG recordings (C). Amplitudes of $10 \mathrm{~Hz}$ flicker ERGs ( $\mathrm{n}=10$ mice) (D). Amplitudes of $20 \mathrm{~Hz} E R G$ at 8 months ( $n=10$ mice) (E). Cone density on flat-mounted retinas ( $n=8$ eye, one per mouse) $(\mathbf{F})$. Representative images of 8 -month-old mouse retinas labeled with OPN1MW and OPN1SW antibodies for cone density measurements (G). Lower panel: 40 fold amplification of the * area of each retina. Scale 1.25 x: $1 \mathrm{~mm} ; 40$ x: $25 \mu \mathrm{m}$. ERG photopic or flicker data are the average of both eyes of each mouse. Error bars correspond to SEM: ${ }^{*}: p<0.05 ;{ }^{* *}: p<0.01$ and ${ }^{* * *}: p<0.001$.

\section{Figure 3.}

Cone function and viability of cones of the [Cone-Cre:Nxn/1cd/cd] mice are reduced after exposure of young animals to hyperoxia. Representative photopic ERG recordings (A). Amplitudes of photopic ERGs (Normoxia: $n=8$ mice; Hyperoxia: $n=18$ mice) (B). Representative $10 \mathrm{~Hz}$ flicker ERG recordings (C). Amplitudes of $10 \mathrm{~Hz}$ flicker ERGs (Normoxia: $\mathrm{n}=8$ mice; Hyperoxia: $n=20$ mice) (D). Cone density on flat-mounted retinas (Normoxia: $n=7$ eyes, one per mouse; Hyperoxia: $n=12$ eyes, one per mouse) (E). Representative images of retina labeled with OPN1MW and OPN1SW antibodies for cone density measurements. Retinas of 1 month old mice under $21 \%$ normoxia. Retina of 1 month old mice after 1 week $75 \%$ hyperoxia treatment. Lower panel: 40 fold amplification of the * area of each retina $(\mathbf{F})$. Scale $1.25 \mathrm{x}$ : 1 $\mathrm{mm} ; 40 \mathrm{x}: 25 \mu \mathrm{m}$. Normoxia: $21 \% \mathrm{O}_{2}$; hyperoxia: $75 \% \mathrm{O}_{2}$. ERG photopic or flicker data are the average of both eyes of each mouse. Error bars correspond to SEM: ${ }^{*}: p<0.05,{ }^{* *}: p<0.01$, and $^{* * * *}: p<0.0001$. 


\section{Figure 4.}

Oxidative damage in retinal sections. Oxidative stress was induced in retinal cells after 1 week under $75 \% \mathrm{O}_{2}$. Immunostaining of retinal sections of $\mathrm{Nxn} / 1+/+, \mathrm{N} x n / 1-/-$, [Cone-Cre:Nxn/1+/+] and [Cone-Cre:Nxnl1cd/cd] mice at 1 month: DAPI (blue), PNA (green) and 4-hydroxynonenal $(\mathrm{HNE}, \mathrm{red})(\mathbf{A})$, or acrolein (red) (B). Scale bar: $20 \mu \mathrm{m}$.

\section{Figure 5.}

Cones express the thioredoxin RdCVFL and not the trophic factor RdCVF. Image of a representative portion of the retina injected with AAV2-7m8-OPN1L/MW-GFP (green) and labeled by anti-OPN1MW antibodies (red and green) (A). Scale bar, $20 \mu \mathrm{m}$. Current responses to voltage steps of a GFP-positive cell by stepping the membrane potential from $-100 \mathrm{mV}$ to $+40 \mathrm{mV}$ in $10 \mathrm{mV}$ increments (B). Current-voltage (I-V) curve of the same cell (C). Single cell RT-PCR of negative control ( 0 , first lane), cone (second lane) and positive control (+, third lane) (D). RT-PCR analysis of twelve clamped cones from a total of retinas of 6 individual mice (E).

\section{Figure 6.}

RdCVFL protects cones against oxidative stress. Amplitudes of photopic ERGs (A, n=18; $\mathbf{B}$, $\mathrm{n}=11$ ). Representative photopic ERG recordings (C). ERG recordings of the corresponding controlateral uninjected eye (D). Amplitudes of $10 \mathrm{~Hz}$ flicker ERGs (E, $n=21 ; \mathbf{F}, n=8)$. Representative $10 \mathrm{~Hz}$ flicker ERG recordings (G). Cone density on flat-mounted retinas $(\mathrm{n}=8)$ (H). Representative images of retina labeled with PNA for cone density measurements. Lower 
panel: 40 fold amplification of the * area of each retina. Scale $1.25 X: 1 \mathrm{~mm} ; 40 X: 25 \mu \mathrm{m}(\mathrm{H}) . \mathrm{n}=$ number of injected eyes, one per mouse. Grey bars: [Cone-Cre:Nxn/1+/+], black bars: [ConeCre:Nxn/1-/-] mice. Error bars correspond to SEM: ${ }^{*}: p<0.05,{ }^{* *}: p<0.01,{ }^{* * *}: p<0.001$ and $* * * *: p<0.0001$.

\section{Figure 7.}

Cone-RdCVFL is implicated in cone survival. Luciferase activity after transient co-transfection of $200 \mathrm{ng}$ miRdCVFL plasmid or its scrambled control with the RdCVFL-Renilla luciferase reporter plasmid in HEK293 cells (A). The number of live chicken cones $(n=30)$ one week after electroporation with scrambled control (200 ng) or miRdCVFL (200 ng and $400 \mathrm{ng})$ (B). Image of the cone-enriched culture cells electroporated with the RFP reporter (C). Error bars correspond to standard deviation in $(A)$ and SEM in $(B):{ }^{*}: p<0.05,{ }^{* *}: p<0.01,{ }^{* *}$ : $p<0.001$ and $^{* * * *}: p<0.0001$

\section{Figure 8.}

RdCVFL expression in cones of the rd10 mice results in their protection. Representative photopic ERG recordings (A). ERG recordings of the corresponding controlateral uninjected eye (B). Amplitudes of photopic ERGs ( $n=7)(C)$. Representative $10 \mathrm{~Hz}$ flicker ERG recordings (D). Amplitudes of $10 \mathrm{~Hz}$ flicker ERGs $(n=7)(E)$ and those of $20 \mathrm{~Hz}$ flicker ERGs $(n=7)(F)$ at PN40. Cone density on flat-mounted retinas $(n=7)(\mathbf{G})$. Representative images of retina labeled with OPN1MW and OPN1SW antibodies for cone density measurements. Lower panel: 40 fold amplification of the ${ }^{*}$ area of each retina $(\mathbf{H})$. Scale 1.25X: $1 \mathrm{~mm} ; 40 \mathrm{X}: 25 \mu \mathrm{m} . \mathrm{n}=$ number of injected eyes, one per mouse. wt: C57BL6/N strain. Error bars correspond to SEM: *: $p<0.05$. 


\section{Figure 9.}

Theoretical effects of reactive oxygen species on aerobic glycolysis. RdCVF: rod-derived cone viability factor, RdCVFL: rod-derived cone viability factor long, red, ox: oxidized, ${ }^{\text {red: }}$ reduced, BSG1: basigin 1, GLUT1: facilitated glucose transporter 1 (SLC2A1), HEX: hexokinase, GPI: glucose phosphate isomerase, PFK: phosphofructokinase, ALDO: aldolase, GAPDH: glyceraldehyde-3-phosphate dehydrogenase, TPI: triose phosphate isomerase, PGK: phosphoglycerate kinase, PGM: phosphoglycerate mutase, ENO: enolase, PKM: pyruvate kinase, LDH: lactate dehydrogenase, MCT1: facilitated lactate transporter 1 (SLC16A1), G/c: glucose, G6P: glucose-6-phosphate, G6PDH: Glucose-6-phosphate dehydrogenase, PGD: 6phosphogluconate dehydrogenase, R5P: Ribulos-5-phosphate, PPP: pentose phosphate pathway, PEP: phosphoenol pyruvate, NADPH: nicotinamide adenine dinucleotide phosphate, TrxRs: thioredoxin reductases, PYR: pyruvate, Lact: lactate, OXPHOS: oxidative phosphorylation, ROS: reactive oxygen species.

Supplementary Figure 1. Fluorescence activated cell sorting (FACS). Retinal cells from the [Cone-Cre:Nxnl1cd/cd] mouse were labeled with rabbit anti-OPN1MW antibodies (C, E) or rabbit anti-lgG (B, D), followed with R-phycoerythrin. Two types of cells were detected by FACS (A). All type1 cells (C) exhibit low fluorescence intensity as the negative control (B). In type 2 cells, the $2 \%$ of cells that contain higher fluorescent intensity $(E)$ than their negative control (D) were collected and analyzed. 
Supplementary Figure 2. Representative $20 \mathrm{~Hz}$ ERG recordings at 8 months (A). Representation of the b-wave amplitude and the implicit time from ERG photopic traces (B). Implicit time of photopic ERGs ( $\mathrm{n}=10$ mice) Blue: Cone-Cre-Nxn/1+/+, Red: one-Cre-Nxn/1cd/cd and green: Nxn/1-/- (C). Cone density on flat-mounted retinas at 1 month $(n=7) 4$ months $(n=5)$ and 8 months $(n=7)$. n: eye, one per mouse. Error bars correspond to SD: ${ }^{*}: p<0.05,{ }^{* *}: p<$ 0,01 (D). Representative images from flat-mounted retinas at high resolution (x100) of 4-months old mice labeled with a mixture of S- and M-antibodies. Scale bars $60 \mu \mathrm{m}(\mathbf{E})$.

Supplementary Figure 3. Representative $20 \mathrm{~Hz}$ ERG recordings at 1 month (A). Amplitudes of $20 \mathrm{~Hz}$ ERGs at 1 month (normoxia: $\mathrm{n}=8$ mice; hyperoxia: $\mathrm{n}=20$ mice) (B). Representative traces of scotopic ERG after $3 \times 10-1 \mathrm{~cd} . \mathrm{s} / \mathrm{m}^{2}$ flash intensity at 1 month of age (C). Scotopic a-wave amplitudes (D, normoxia $n=5$ mice; hyperoxia $+/+, n=6$ mice; $c d / c d n=8$ mice). Scotopic b-wave amplitude. The asterisk indicates that the values are statistically different at $3 \times 10^{-1} \mathrm{~cd} . \mathrm{s} / \mathrm{m}^{2}$ normoxia $\mathrm{n}=5$ mice; hyperoxia $+/+, \mathrm{n}=6$ mice; $c d / c d \mathrm{n}=8$ mice). Representative images of the OCT (F). Outer nuclear layer thickness along the temporo-nasal axis (G, normoxia $n=5$ mice; hyperoxia +/+, $\mathrm{n}=7$ mice; $c d / c d \mathrm{n}=9$ mice). Outer nuclear layer thickness along the dorso-ventral axis $(H$, normoxia $n=5$ mice; hyperoxia $+/+, n=7$ mice; $c d / c d n=9$ mice). Error bars correspond to SEM: ${ }^{* *}: p<0.01{ }^{* * *}: p<0.001$ and ${ }^{* * *}: p<0.0001$.

Supplementary Figure 4. Amplitudes of $20 \mathrm{~Hz}$ flicker ERGs $(\mathbf{A}, \mathrm{n}=20 ; \mathbf{B}, \mathrm{n}=8)$ at PN40. Representative traces of $20 \mathrm{~Hz}$ flicker ERGs at PN40 (C). Implicit time of photopic ERGs ( $\mathrm{n}=8$ mice) (D). $\mathrm{n}=$ number of injected eyes, one per mouse. Correlation between the photopic and $10 \mathrm{~Hz}$ flicker ERG. Data plotted for 53 [Cone-Cre:Nxn/1cd/cd] measurements of photopic versus flicker $10 \mathrm{~Hz}$ ERG are giving a Pearson correlation coefficient of 0.6354 . The coefficient is 
0.5089 when comparing photopic to flicker $20 \mathrm{~Hz} . \mathrm{n}=$ number of injected eyes, one per mouse. Error bars correspond to SEM: ${ }^{*}: p<0.05,{ }^{* * *}: p<0.0001$.

Supplementary Figure 5. Representative traces of $20 \mathrm{~Hz}$ flicker ERGs (A). Correlation between the photopic and $10 \mathrm{~Hz}$ flicker ERG. Data plotted for $42 \mathrm{rd} 10$ measurements of photopic versus flicker $10 \mathrm{~Hz}$ ERG are giving a Pearson correlation coefficient of 0.503 . The outlier in red (11360) corresponds to a non-injected contralateral eye (B). The coefficient is 0.544 when comparing photopic to flicker $20 \mathrm{~Hz}$. 
A

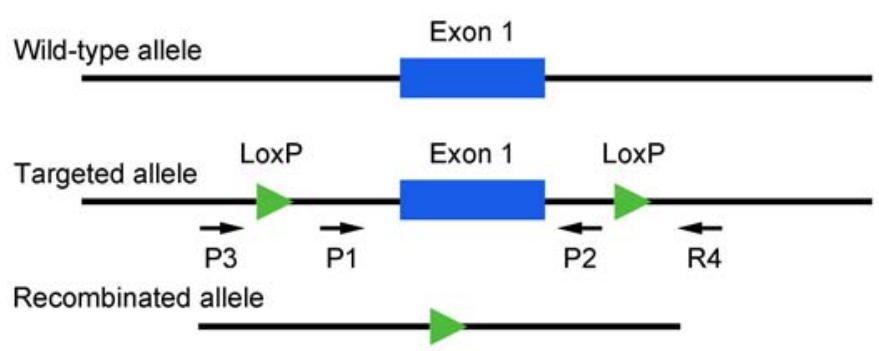

B

\section{C}
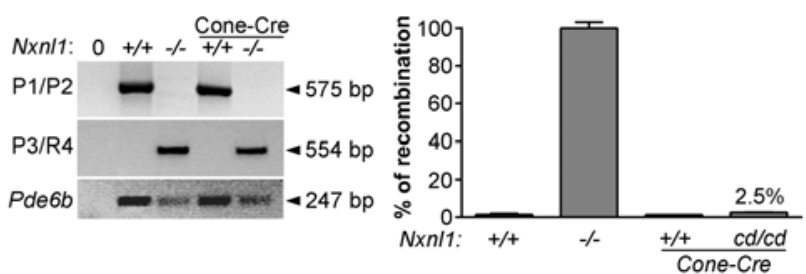

D
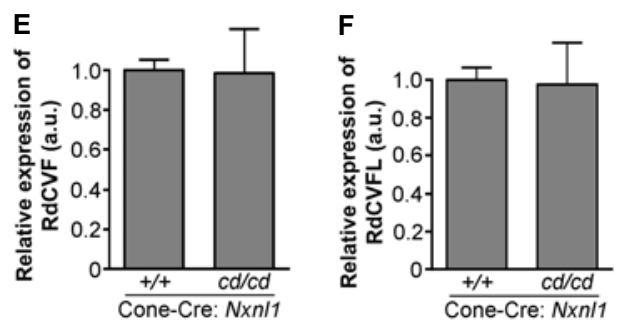

G

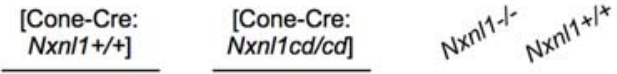

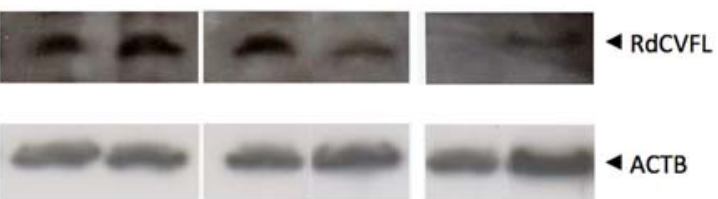


A
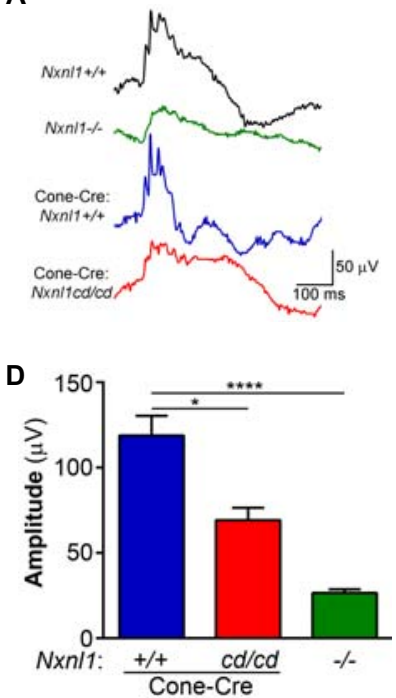

G

Nxnl1+/+
B
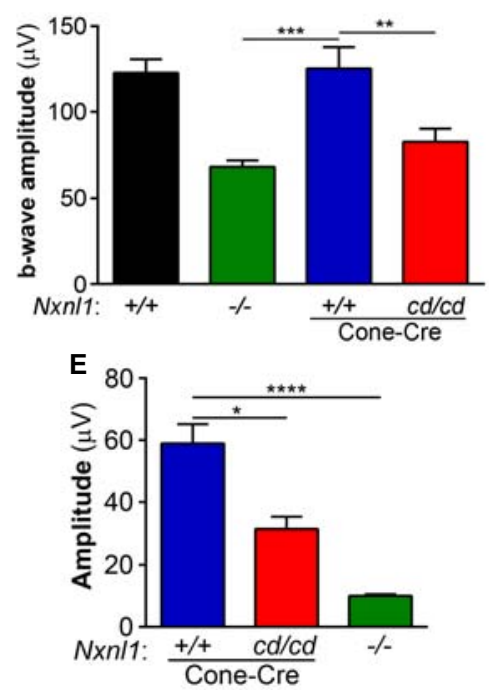

Nxn/1-/-

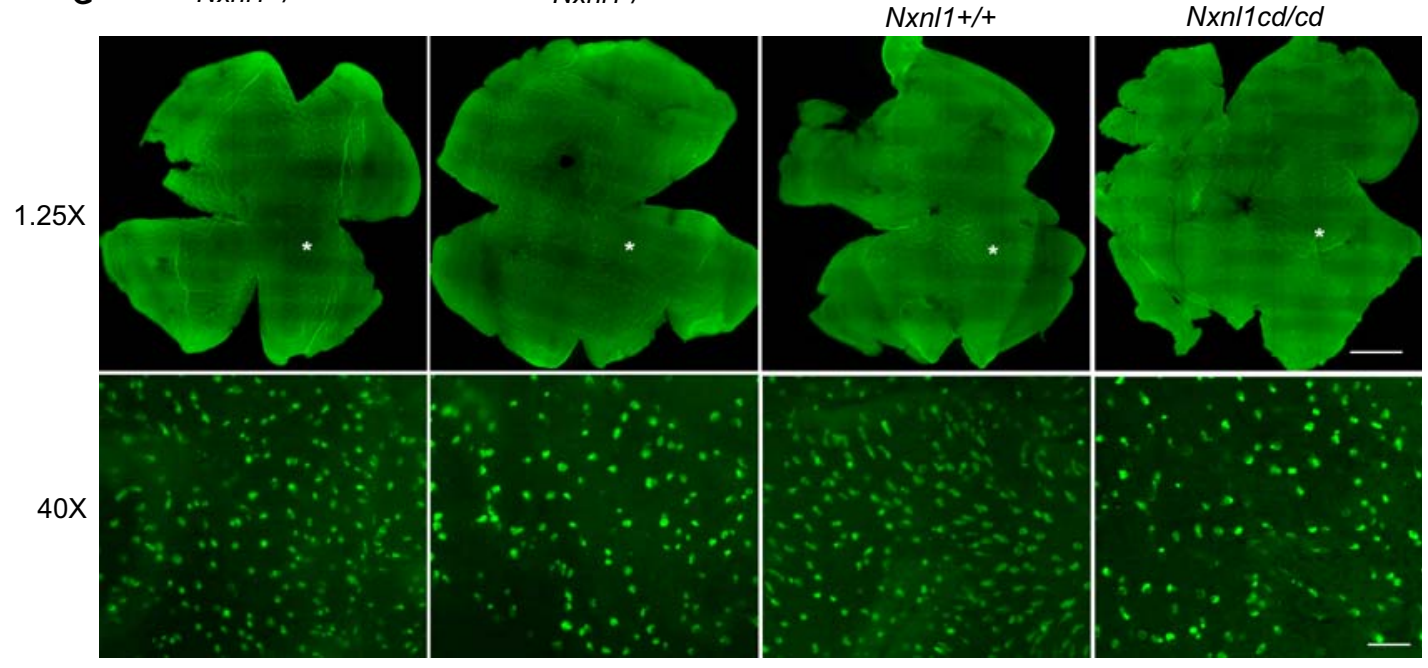

C

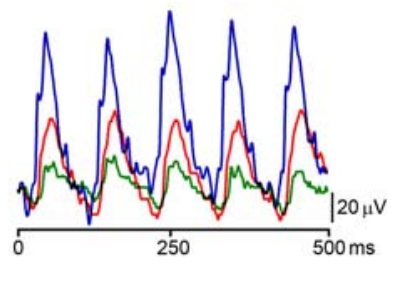

F

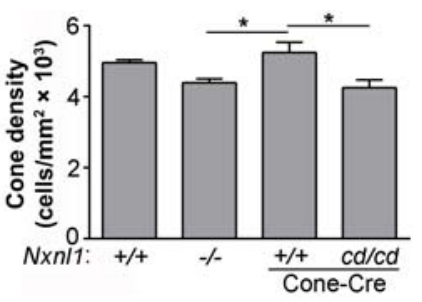

Cone-Cre: Nxn/1cd/cd 
A

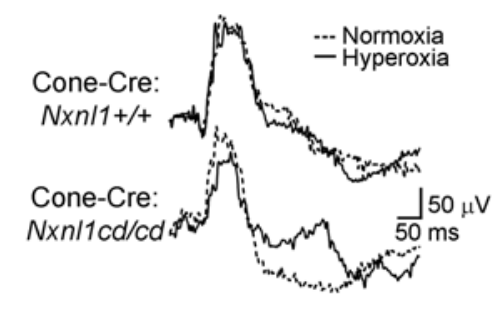

Cone-Cre:
Nxnl1+/+

$\left.\begin{array}{c}\text { Cone-Cre: } \\ \text { Nxnl1cd/cd }\end{array}\right]$

... Normoxia - Hyperoxia

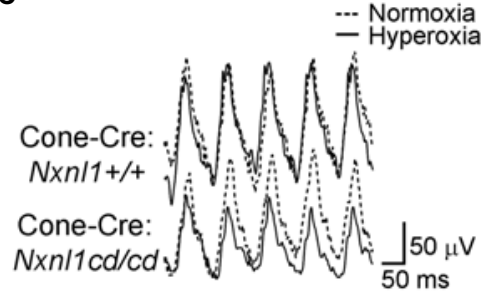

B

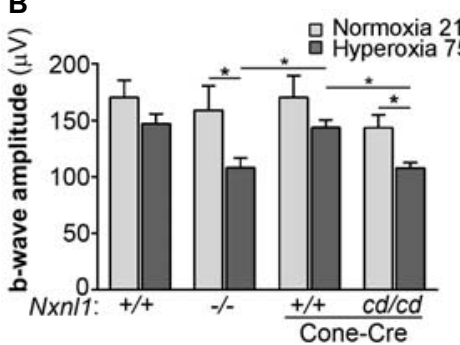

D $200 \quad \begin{array}{r}\text { a Normoxia } 21 \% \\ \text { Hyperoxia } 75 \%\end{array}$

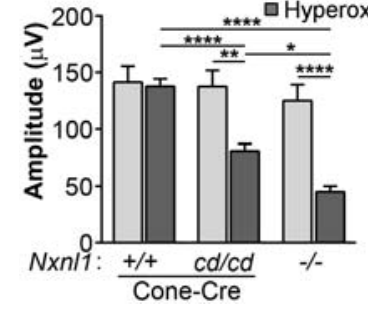

F

Nxnl1+/+

NxnI1-/-

Cone-Cre: Nxnl1+/+

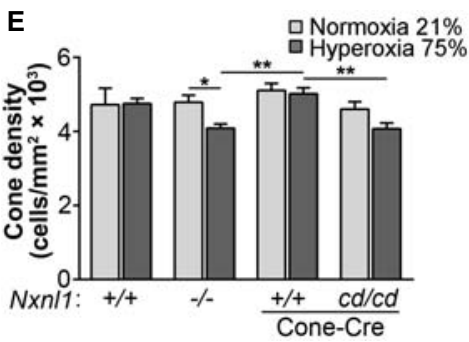

$1.25 X$

$40 X$
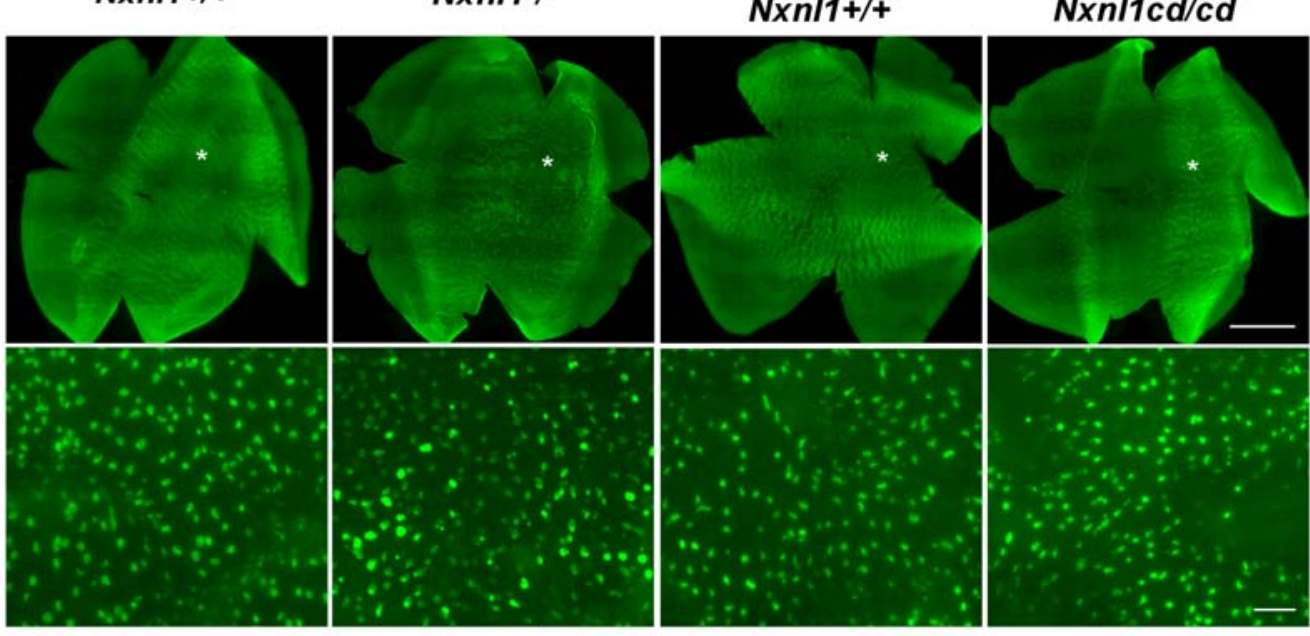

$1.25 X$
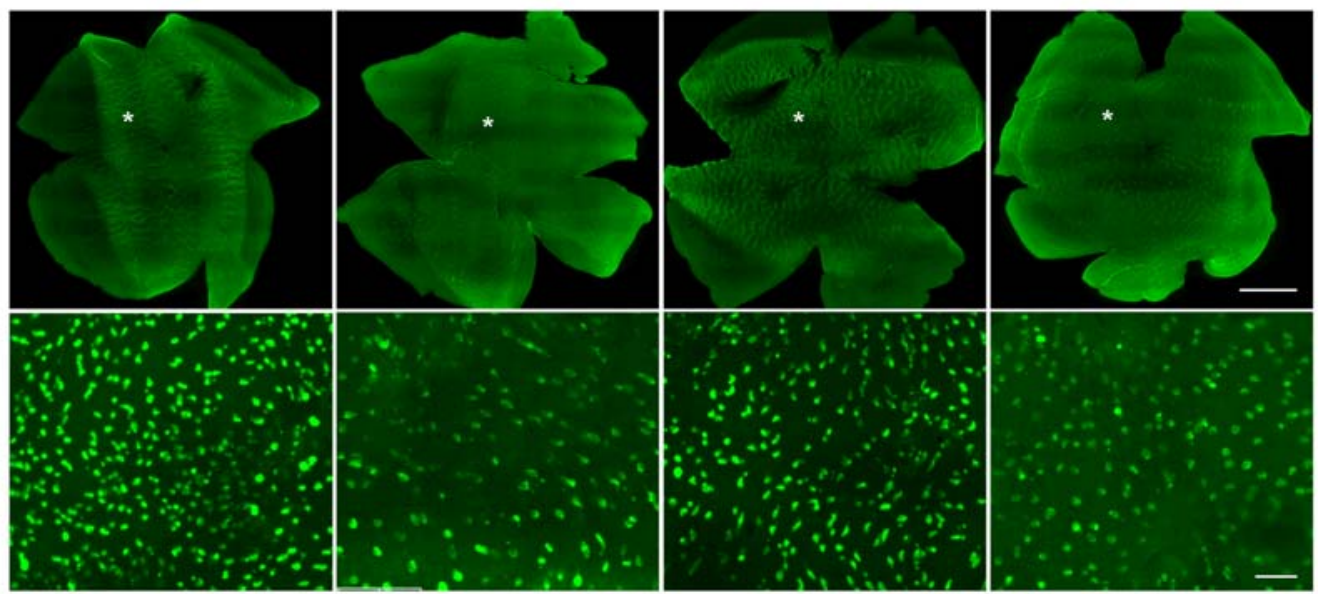


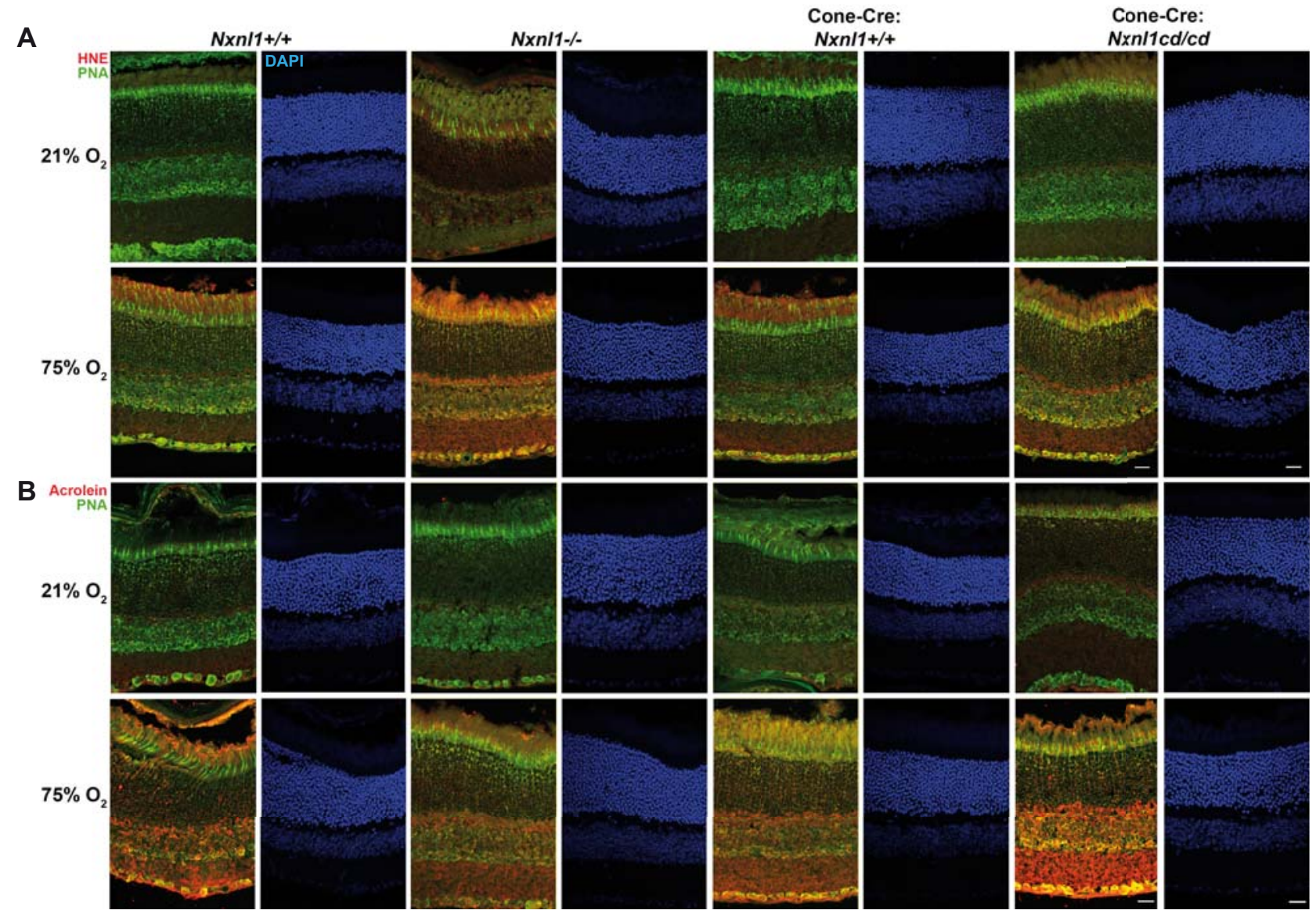



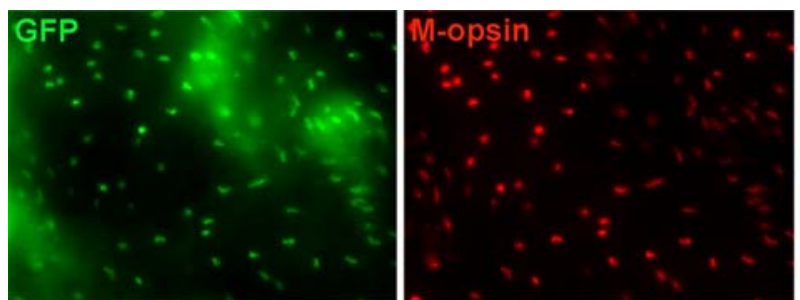

B

C
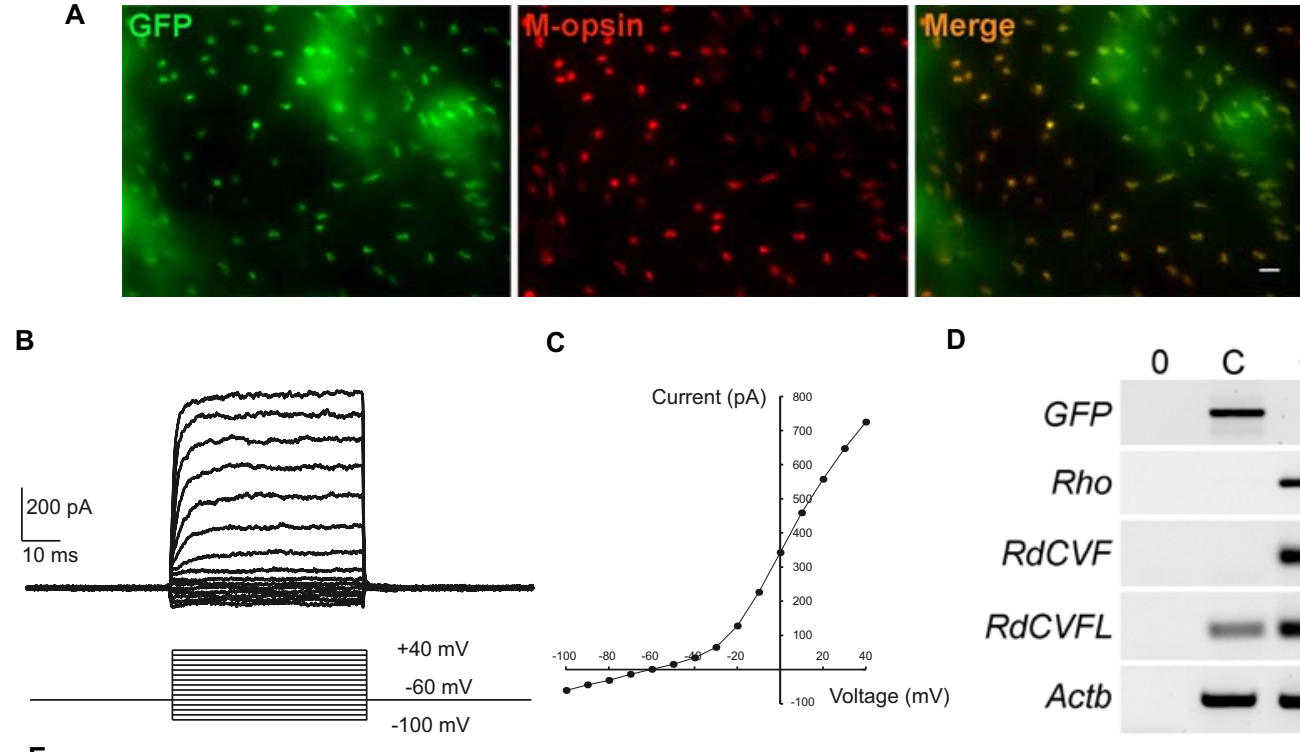

D
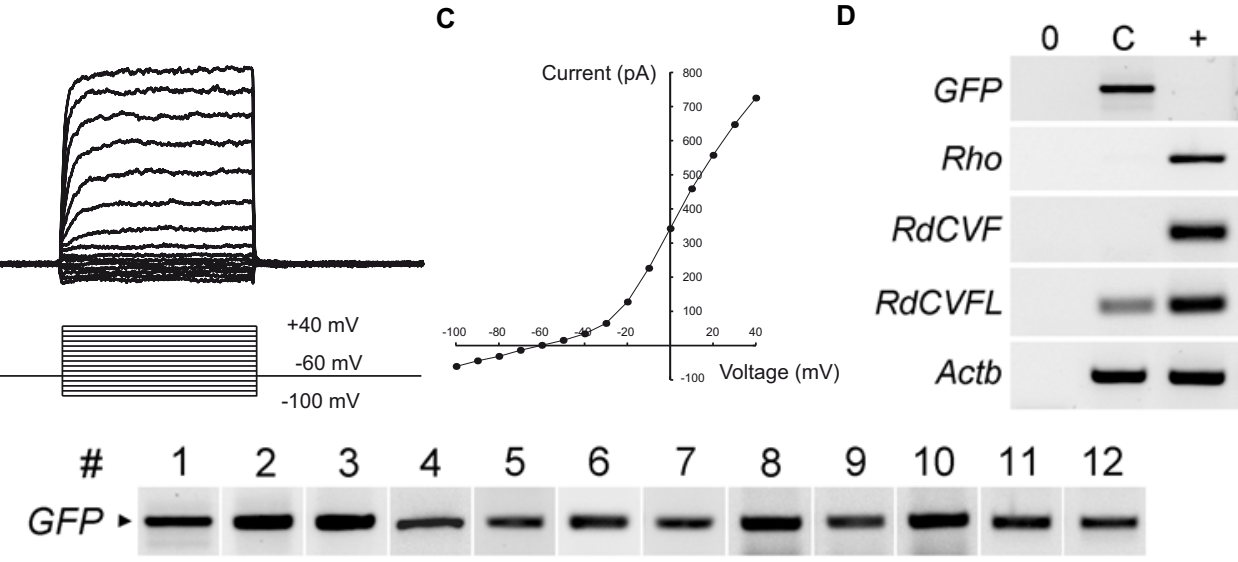

RdCVF .

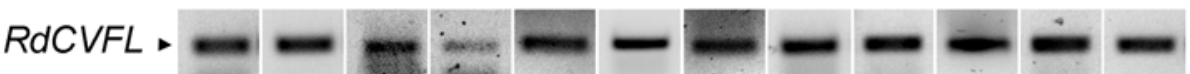


A

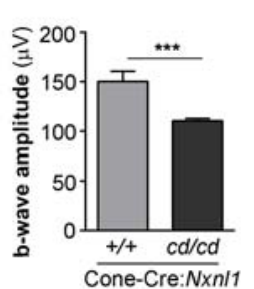

E

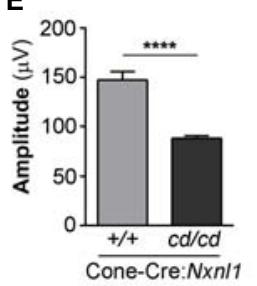

B
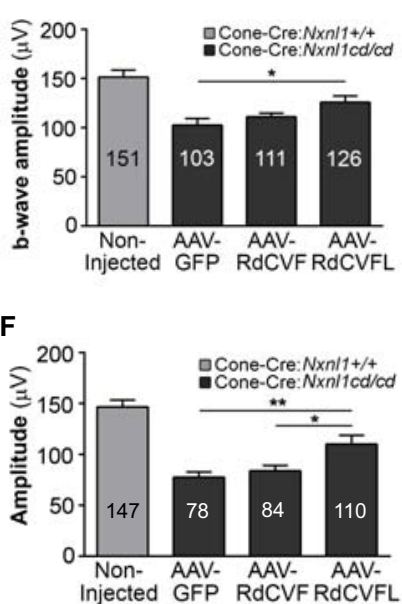

C

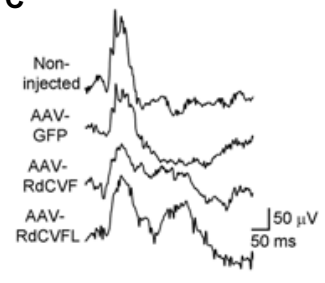

G

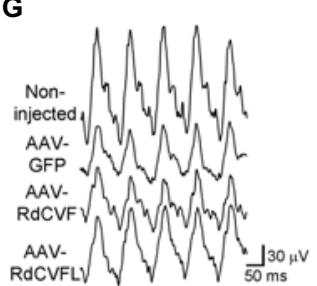

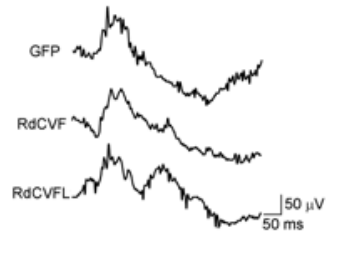

H

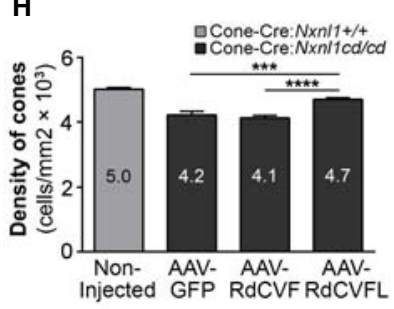

I

Cone-Cre:Nxnl1+/+

Non injected

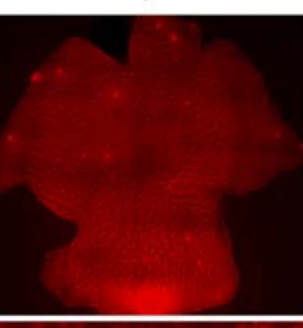

AAV2.8-GFP

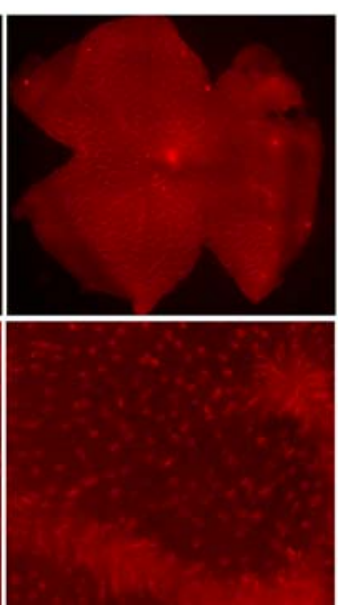

Cone-Cre:Nxnl1cd/cd

AAV2.8-RdCVFL

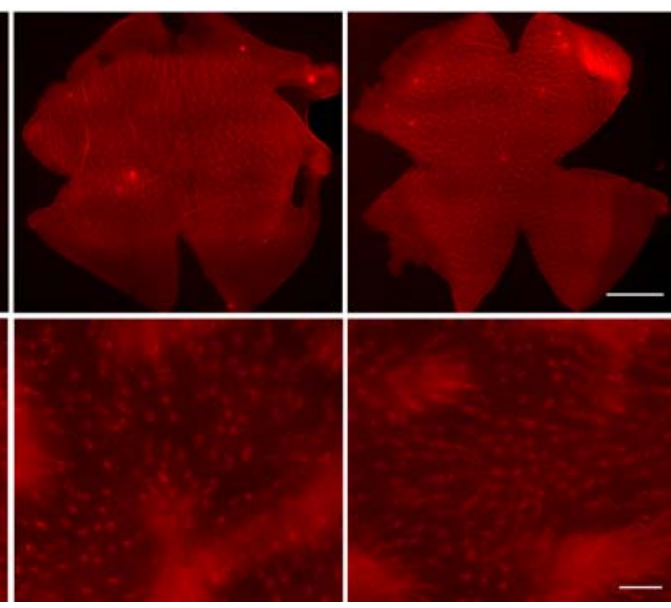


A

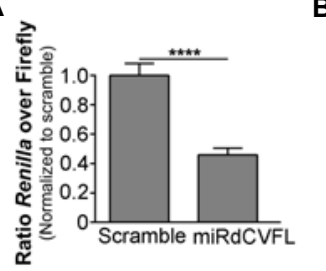

B

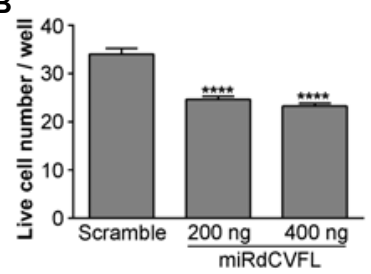

C

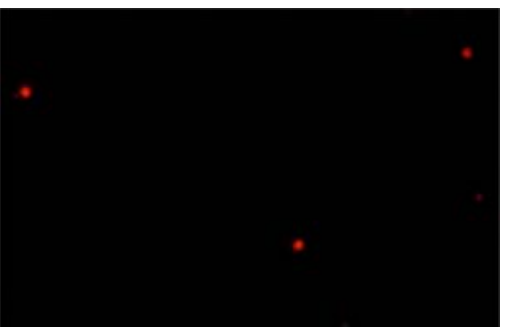




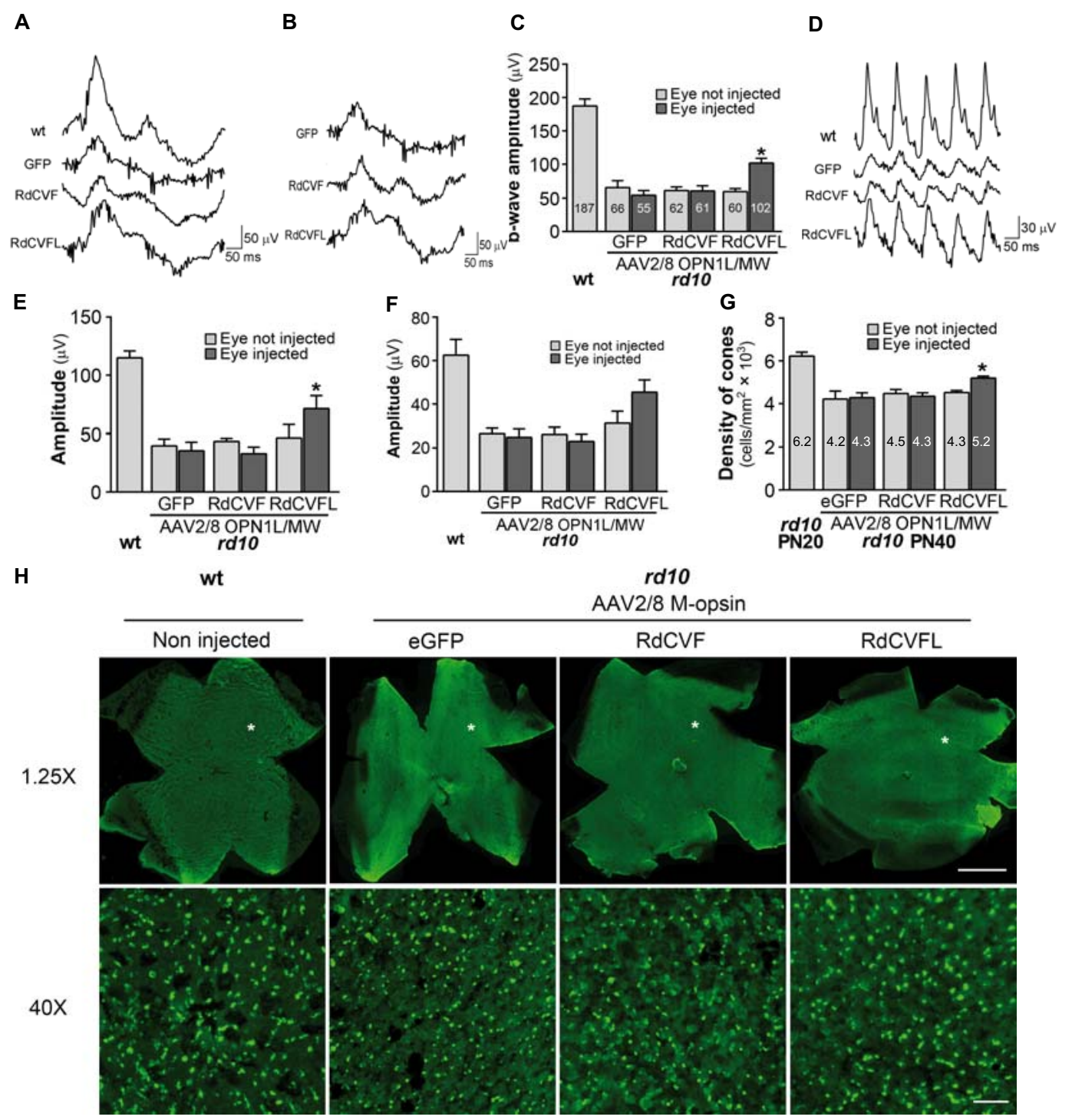




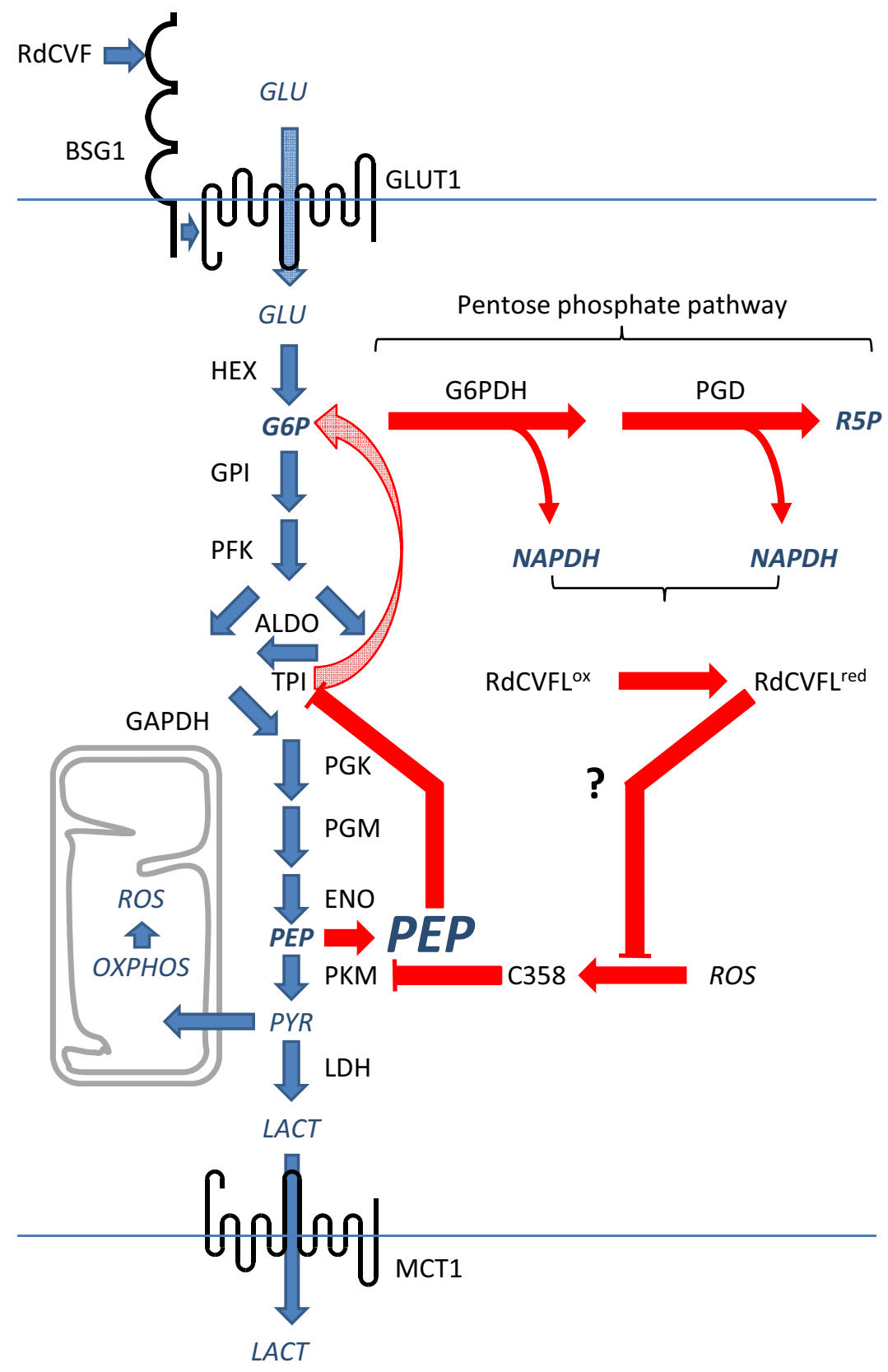



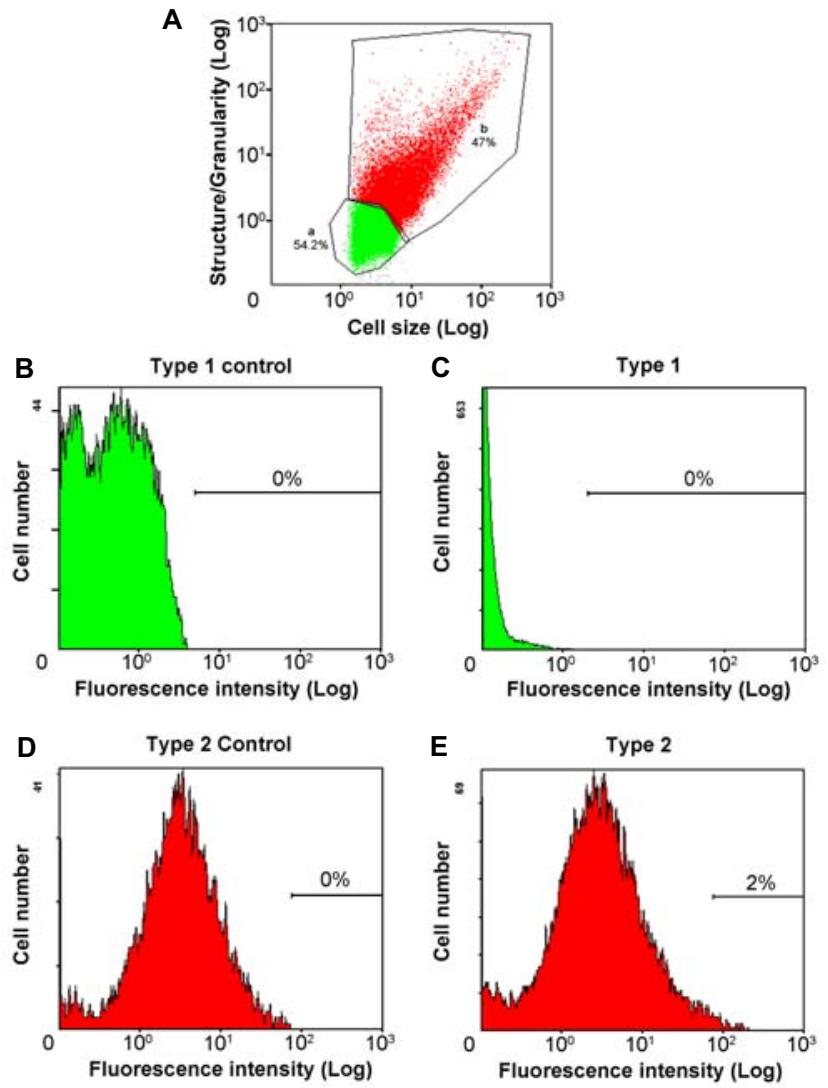
A

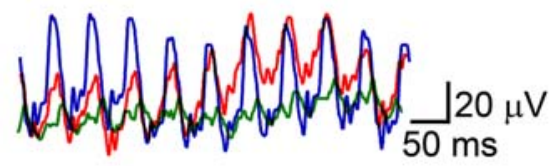

B

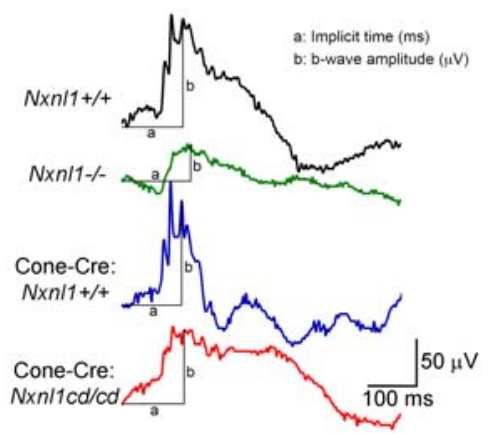

C

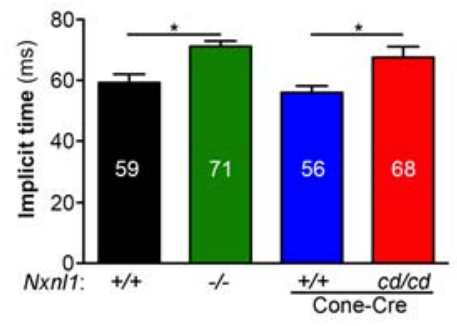

D
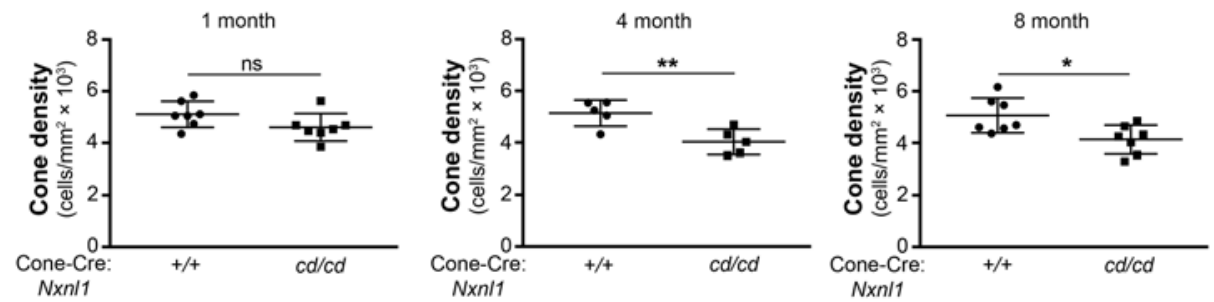

E

Cone-Cre:Nxn/1+/+

Dorso-temporal

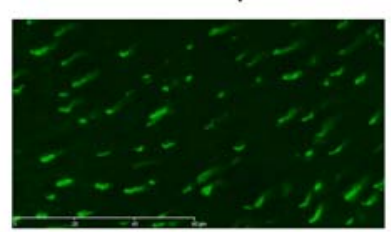

Ventro-temporal

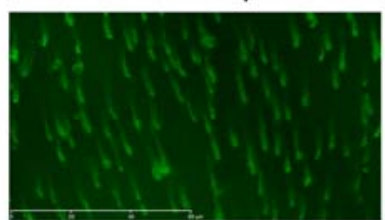

Dorso-nasal

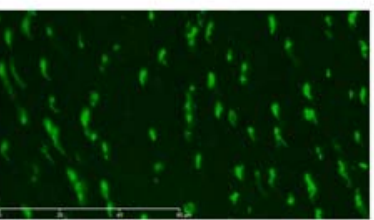

Ventro-nasal

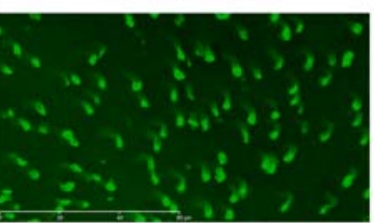

Cone-Cre:Nxn/1cd/cd

Dorso-temporal

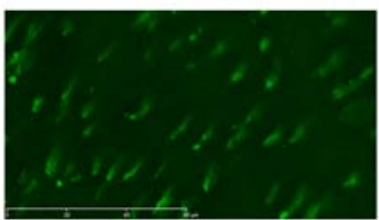

Ventro-temporal

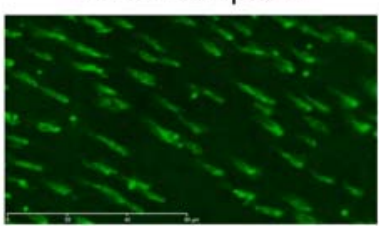

Dorso-nasal

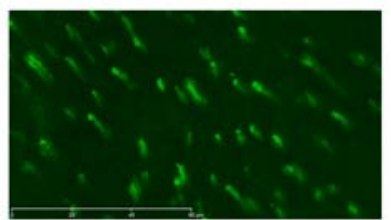

Ventro-nasal

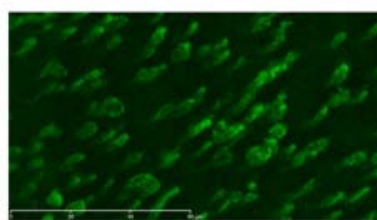


A

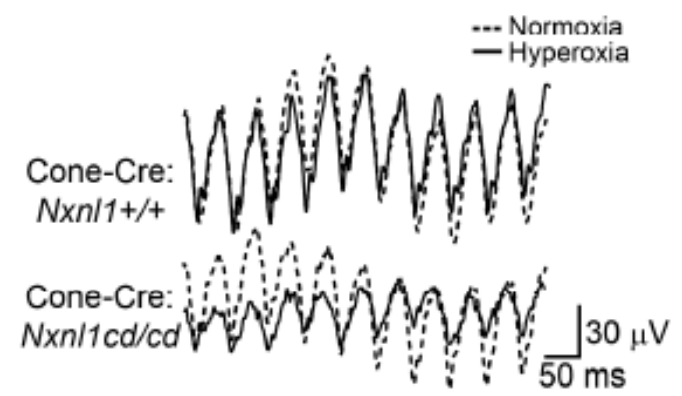

B

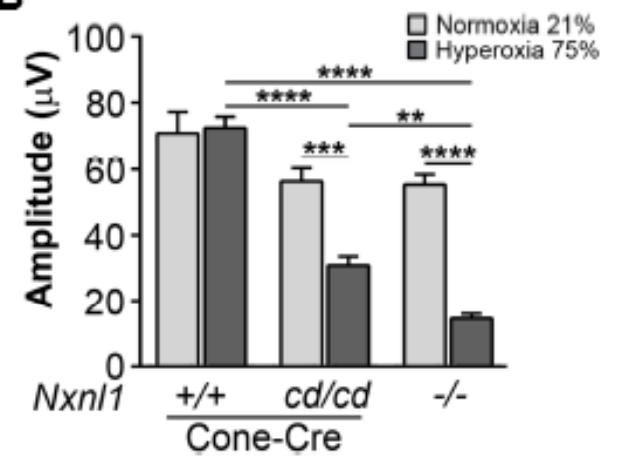

C

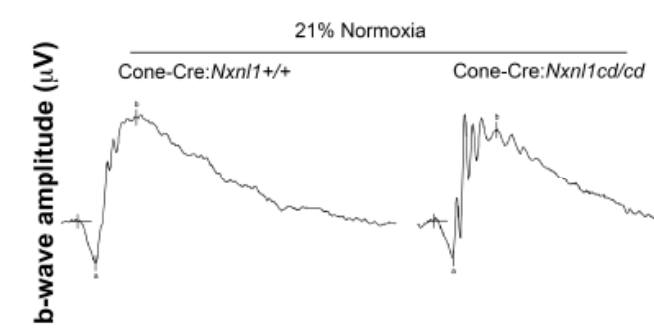

D

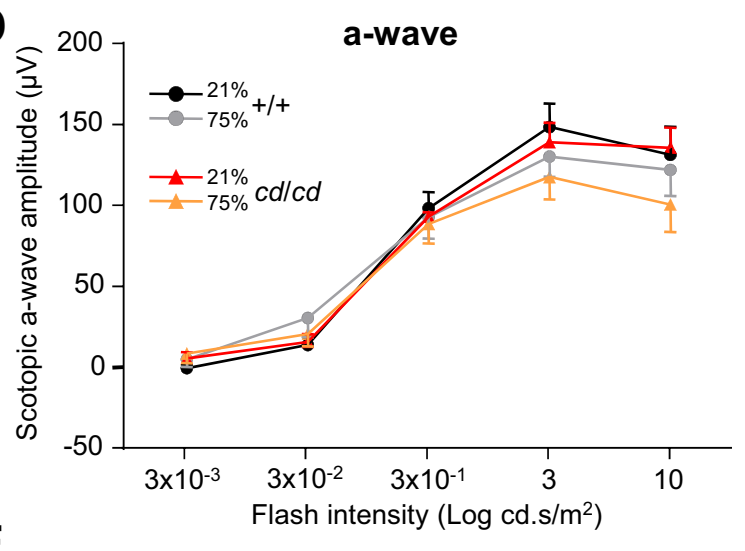

E

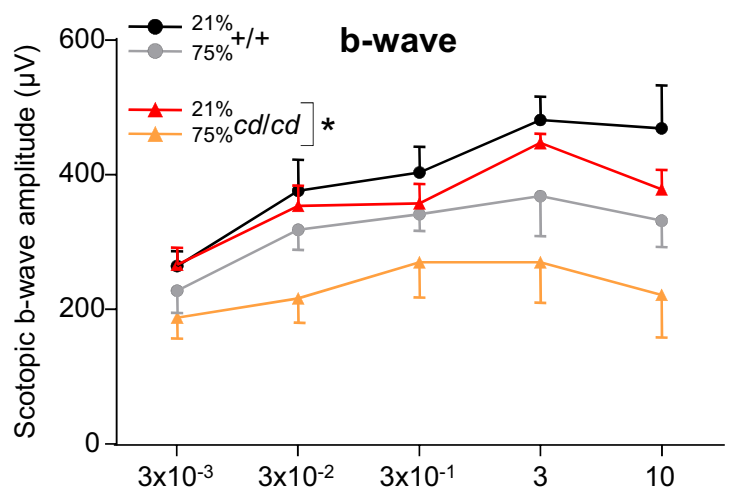

G

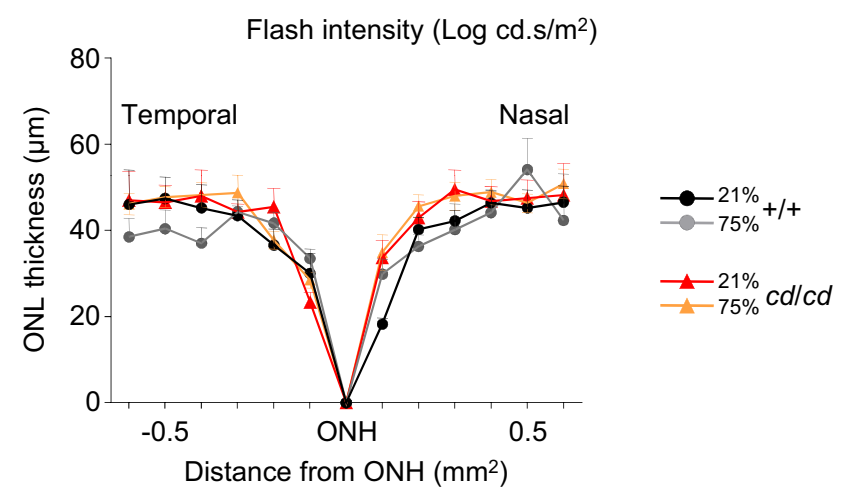

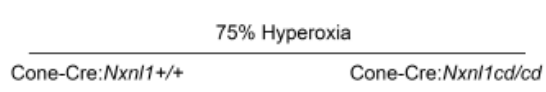
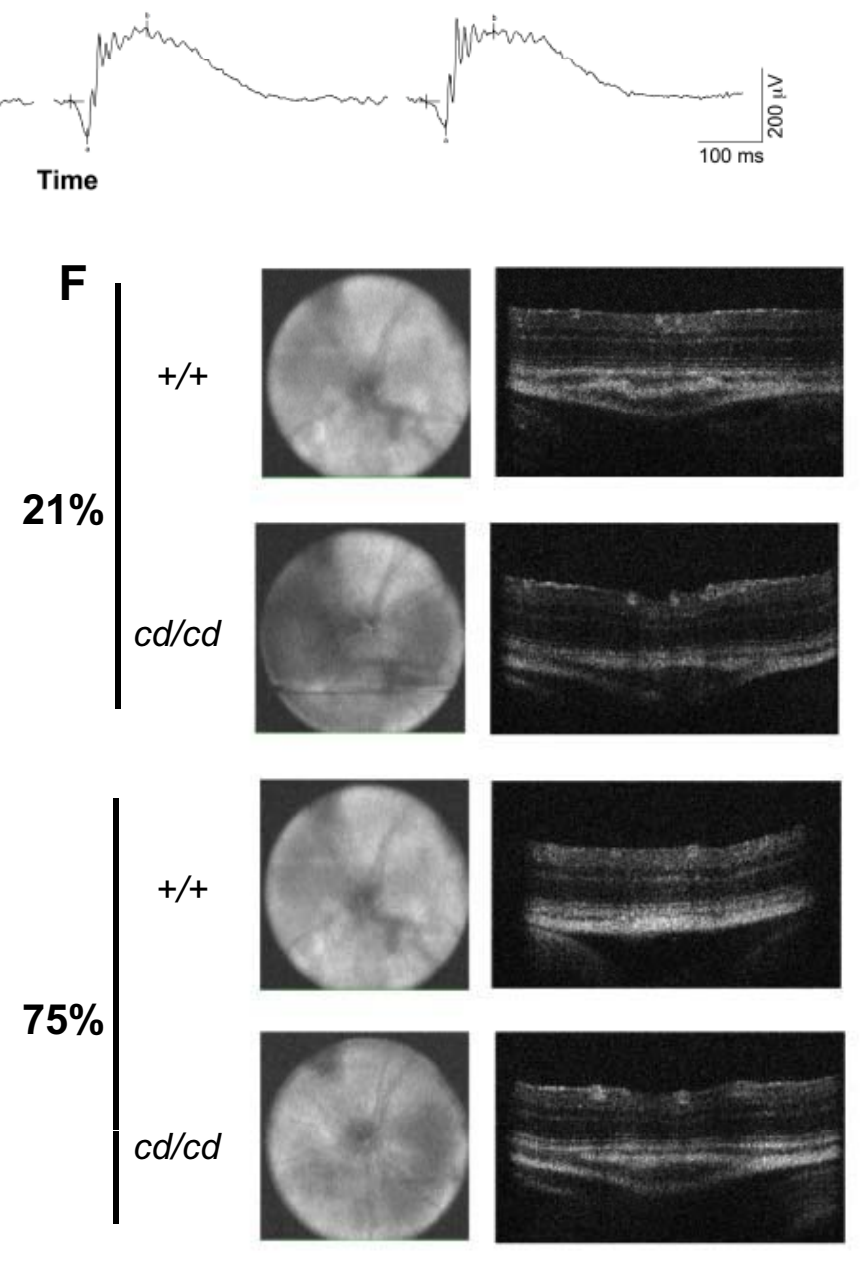

H

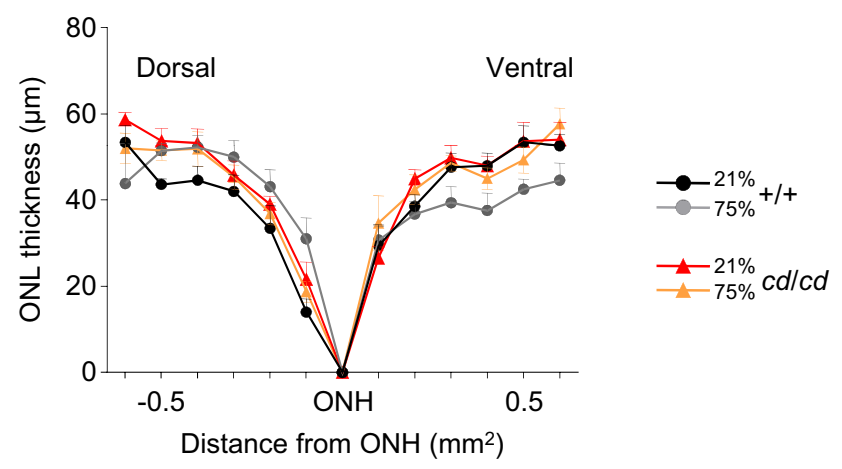



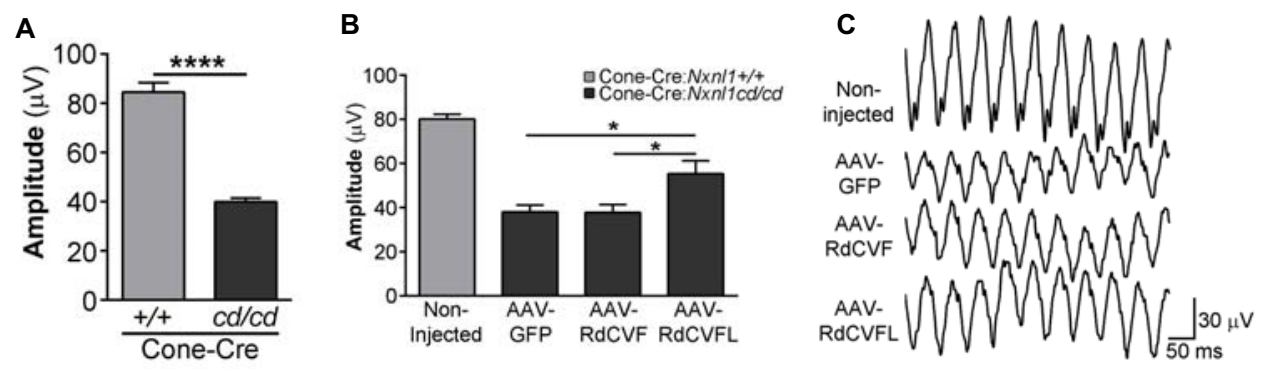

D

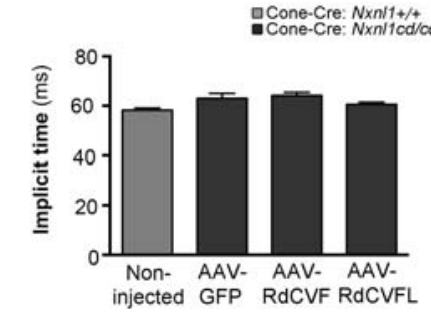

E

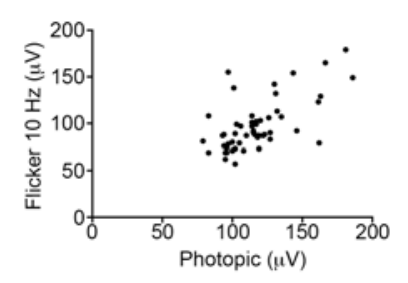


A

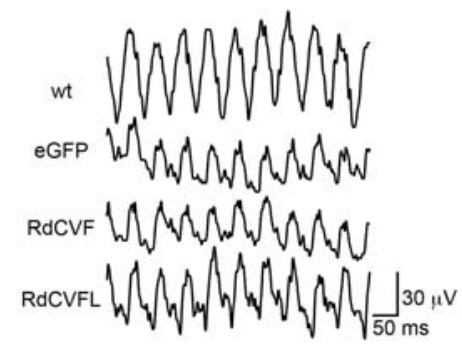

B

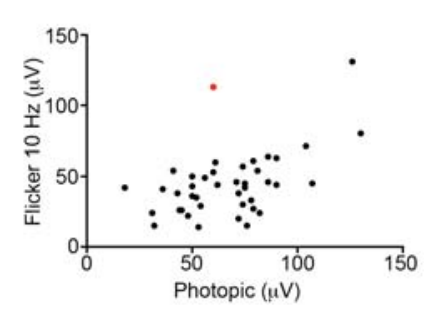

